\title{
Residual properties of automorphism groups of (relatively) hyperbolic groups
}

\author{
Gilbert Levitt
}

Ashot Minasyan

\begin{abstract}
We show that $\operatorname{Out}(G)$ is residually finite if $G$ is one-ended and hyperbolic relative to virtually polycyclic subgroups. More generally, if $G$ is one-ended and hyperbolic relative to proper residually finite subgroups, the group of outer automorphisms preserving the peripheral structure is residually finite. We also show that $\operatorname{Out}(G)$ is virtually residually $p$-finite for every prime $p$ if $G$ is one-ended and toral relatively hyperbolic, or infinitelyended and virtually residually $p$-finite.
\end{abstract}

20F67, 20F $28 ; 20 \mathrm{E} 26$

\section{Introduction}

A group $G$ is residually finite if, given any $g \neq 1$, there exists a homomorphism $\varphi$ from $G$ to a finite group such that $\varphi(g) \neq 1$. Residual finiteness is an important property of groups. It is equivalent to the statement that $G$ embeds into its profinite completion. Well known theorems of Mal'cev claim that finitely generated residually finite groups are Hopfian, and finitely presented residually finite groups have solvable word problem. Many groups are known to be residually finite (in particular, finitely generated linear groups), but it is a big open question whether all (Gromov) hyperbolic groups are residually finite.

It is a standard and classical fact [5] that the automorphism group $\operatorname{Aut}(G)$ is residually finite if $G$ is finitely generated and residually finite, but this is not true for the outer automorphism group $\operatorname{Out}(G)$. Indeed, any finitely presented group may be represented as $\operatorname{Out}(G)$ with $G$ finitely generated and residually finite [8].

A special case of our main theorem is:

Corollary 1.1 If $G$ is one-ended, and hyperbolic relative to a family $\mathcal{P}=\left\{P_{1}, \ldots, P_{k}\right\}$ of virtually polycyclic groups, then $\operatorname{Out}(G)$ is residually finite.

This is new even if $G$ is a torsion-free hyperbolic group. Work by Sela implies that a finite index subgroup of $\operatorname{Out}(G)$ is virtually a central extension of a free abelian group by a direct product of mapping class groups (see [23]). Though mapping class groups are known to be residually finite following work by Grossman based on conjugacy separability [2, 15], this is not enough to deduce residual finiteness of $\operatorname{Out}(G)$ because the extension may fail to split (i.e., be a direct product); see the example discussed in the introduction of [23].

To complement Corollary 1.1, recall that $\operatorname{Out}(G)$ is residually finite if $G$ is residually finite and has infinitely many ends [28]. On the other hand, $\operatorname{Out}\left(G * F_{2}\right)$ contains $G$ (with $F_{2}$ the free group of rank 2), so it is not residually finite if $G$ is not. Thus one-endedness cannot be dispensed with in Corollary 1.1. This also gives a direct way of proving the following fact, which may otherwise be obtained by combining small cancellation theory over hyperbolic groups with the results from [28]. 
Proposition 1.2 The following are equivalent:

(i) Every hyperbolic group is residually finite.

(ii) For every hyperbolic group $G$, the $\operatorname{group} \operatorname{Out}(G)$ is residually finite.

Virtual polycyclicity of the $P_{i}$ 's is used in two ways in Corollary 1.1 (see Subsection 7.6). It ensures that $P_{i}$ is residually finite, and also that automorphisms of $G$ respect the peripheral structure: $P_{i}$ is mapped to a conjugate of a $P_{j}$ (this only holds if no $P_{i}$ is virtually cyclic, but such a restriction causes no loss of generality, see Subsection 4.1). In fact, the peripheral structure is preserved if every $P_{i}$ is small (i.e., it does not contain the free group $F_{2}$ ) or, more generally, NRH (non relatively hyperbolic) - see [27] for a proof and a list of examples of NRH groups.

Since the peripheral structure is not always preserved, we restrict to the $\operatorname{subgroup} \operatorname{Out}(G ; \mathcal{P})$ of $\operatorname{Out}(G)$ defined as the group of classes of automorphisms mapping each $P_{i}$ to a conjugate.

Theorem 1.3 Let $G$ be a group hyperbolic relative to a family of proper finitely generated subgroups $\mathcal{P}=\left\{P_{1}, \ldots, P_{k}\right\}$. If $G$ is one-ended relative to $\mathcal{P}$, and every $P_{i}$ is residually finite, then $\operatorname{Out}(G ; \mathcal{P})$ is residually finite.

Being one-ended relative to $\mathcal{P}$ means that $G$ does not split over a finite group with each $P_{i}$ contained in a vertex group (up to conjugacy). This is weaker than having at most one end.

If every $P_{i}$ is $\mathrm{NRH}$, then $\operatorname{Out}(G ; \mathcal{P})$ has finite index in $\operatorname{Out}(G)$, so we deduce residual finiteness of $\operatorname{Out}(G)$.

The following example shows that it is indeed necessary to assume that all peripheral subgroups $P_{i}$ are residually finite in Theorem 1.3.

Example 1.4 Let $H$ be a torsion-free non-residually finite group with trivial center (such as the Baumslag-Solitar group $B S(2,3))$. Let $K$ be a one-ended torsion-free hyperbolic group (e.g., the fundamental group of a closed hyperbolic surface), and let $\langle k\rangle \leqslant K$ be a maximal cyclic subgroup. Let $G$ be the amalgamated product $(H \times\langle k\rangle) *\langle k\rangle$. Then $G$ is one-ended, torsion-free, and hyperbolic relative to $\mathcal{P}=\{H \times\langle k\rangle\}$ (see [30]). Non-trivial elements $h \in H$ define twist automorphisms (they act as conjugation by $h$ on $H \times\langle k\rangle$ and trivially on $K$ ), which give rise to non-trivial outer automorphisms in $\operatorname{Out}(G ; \mathcal{P})$ because $H$ has trivial center. Thus $H$ can be embedded into $\operatorname{Out}(G ; \mathcal{P})$, and so $\operatorname{Out}(G ; \mathcal{P})$ is not residually finite.

In the last section of the paper we consider residual $p$-finiteness. If $p$ is a prime, $G$ is residually $p$-finite if, given any non-trivial element $g \in G$, there exists a homomorphism $\varphi$ from $G$ to a finite $p$-group such that $\varphi(g) \neq 1$. A group is virtually residually $p$-finite if some finite index subgroup is residually $p$-finite. Evidently residual $p$-finiteness implies residual nilpotence. And if a group is virtually residually $p$-finite for at least two distinct primes $p$, then it is virtually torsion-free.

It is well-known that free groups are residually $p$-finite for any prime $p$, and it is a classical result that a finitely generated linear group is residually $p$-finite for all but finitely many $p$ 's (cf. [42]). Lubotzky [24] proved that for a finitely generated virtually residually $p$-finite group $G$, the group $\operatorname{Aut}(G)$ is also virtually residually $p$-finite, which is a natural analogue of Baumslag's result [5]. Another theorem from [24] states that, if $F$ is a free group of finite $\operatorname{rank}$, then $\operatorname{Out}(F)$ is virtually residually $p$-finite for any prime $p$. The latter result was later extended by Paris [32] to fundamental groups of compact oriented surfaces. The next two theorems generalize these results to certain relatively hyperbolic groups: 
Theorem 1.5 If $G$ is a one-ended toral relatively hyperbolic group, then $\operatorname{Out}(G)$ is virtually residually $p$-finite for every prime number $p$.

Recall that $G$ is called toral relatively hyperbolic if it is torsion-free and hyperbolic relative to a finite family of finitely generated abelian groups. The theorem also applies to groups containing a one-ended toral relatively hyperbolic group with finite index, in particular to virtually torsion-free hyperbolic groups (see Theorem 8.14).

The following statement is a counterpart of Theorem 1.5 in the case when $G$ has infinitely many ends, giving an 'outer' version of Lubotzky's result [24] mentioned above. It is a natural pro- $p$ analogue of $[28$, Thm. 1.5].

Theorem 1.6 If $G$ is a finitely generated group with infinitely many ends and $G$ is virtually residually $p$-finite for some prime number $p$, then $\operatorname{Out}(G)$ is virtually residually $p$-finite.

Recall that limit groups (finitely generated fully residually free groups) are toral relatively hyperbolic $[1,10]$. Residual finiteness of $\operatorname{Out}(G)$ for such a group $G$ was proved by Metafsis and Sykiotis in [26]. Combining Theorems 1.5 and 1.6 gives the following enhancement:

Corollary 1.7 If $G$ is a limit group (finitely generated fully residually free group), then $\operatorname{Out}(G)$ is virtually residually $p$-finite for any prime $p$.

We start the paper by giving a rather quick proof of Corollary 1.1 when $G$ is (virtually) torsion-free hyperbolic, using Sela's description of $\operatorname{Out}(G)$ recalled above as a starting point. The proof of Theorem 1.5 (given in Section 8) uses similar arguments, but in order to prove Theorem 1.3, we have to use different techniques.

Say that a subgroup of a relatively hyperbolic group $G$ is elementary if it is virtually cyclic or parabolic (conjugate to a subgroup of some $P_{i}$ ). As in [19], we consider the canonical JSJ decomposition of $G$ over elementary subgroups relative to $\mathcal{P}$. This is a graph of groups decomposition $\Gamma$ of $G$ such that edge groups are elementary, each $P_{i}$ is conjugate to a subgroup of a vertex group, and $\Gamma$ is $\operatorname{Out}(G, \mathcal{P})$-invariant; moreover, vertex groups are either elementary, or quadratically hanging $(\mathrm{QH})$, or rigid.

In the first step of the proof of Theorem 1.3 (Section 5), we replace each rigid vertex group by a new group which is residually finite and has infinitely many ends. In the second step, we make elementary vertex groups, and edge groups, finite (using residual finiteness of the $P_{i}$ 's). Apart from simple cases, the new graph of groups represents a residually finite group $G^{\prime \prime}$ with infinitely many ends, and so $\operatorname{Out}\left(G^{\prime \prime}\right)$ is residually finite by [28]. Thus we get a homomorphism $\operatorname{Out}(G ; \mathcal{P}) \rightarrow \operatorname{Out}\left(G^{\prime \prime}\right)$ and we show that such homomorphisms "approximate" $\operatorname{Out}(G ; \mathcal{P})$

The second step is easier when $G$ is torsion-free (see Section 6). Torsion brings technical complications, so in its presence we prefer to give a different argument using Dehn fillings [29] and Grossman's method [15]. Sections 7 and 8 are independent of Section 6.

Acknowledgments. We wish to thank the organizers of the 2009 Geometric group theory conference in Bedlewo, where this research was started. The first author was supported in part by ANR-07-BLAN-0141-01 and ANR-2010-BLAN-116-03. The work of the second author was partially supported by the EPSRC grant EP/H032428/1. 


\section{Notations and residual finiteness}

First let us specify some notation.

If $G$ is a group, we denote its center by $Z(G)$. If $H \leqslant G$ is a subgroup, then $Z_{G}(H)$ is its centralizer in $G$. We will write $|G: H|$ for the index of a subgroup $H$ in $G$. For any $g \in G$, we denote by $\tau_{g} \in \operatorname{Aut}(G)$ the inner automorphism given by $\tau_{g}: x \mapsto g x g^{-1}$ for all $x \in G$. If $R \subseteq G$, then $\left\langle\langle R\rangle^{G}\right.$ will denote the normal closure of $R$ in $G$. If $A$ is an abelian group, and $n \geq 1$, we will write $n A=\left\{g^{n} \mid g \in A\right\}$ for the corresponding verbal subgroup of $A$.

Given $\alpha \in \operatorname{Aut}(G)$, we write $\hat{\alpha}$ for its image in $\operatorname{Out}(G)$. We denote by $\operatorname{Aut}(G ; \mathcal{P}) \leqslant$ $\operatorname{Aut}(G)$ the subgroup consisting of automorphisms mapping each $P_{i}$ to a conjugate, and by $\operatorname{Out}(G ; \mathcal{P})$ its image in $\operatorname{Out}(G)$. If every $P_{i}$ is $\mathrm{NRH}$ (e.g., if $P_{i}$ is not virtually cyclic and has no non-abelian free subgroups), then $\operatorname{Out}(G ; \mathcal{P})$ has finite $\operatorname{index} \operatorname{in} \operatorname{Out}(G)($ see $[27$, Lemma 3.2]).

Given a group $G$, the cosets of finite index normal subgroups define a basis of the profinite topology on $G$. This topology is Hausdorff if and only if $G$ is residually finite. A subset $S$ of $G$ is said to be separable if $S$ is closed in the profinite topology. Thus, if $G$ is residually finite, then any finite subset of $G$ is separable.

A subgroup $K \leqslant G$ is closed in the profinite topology if and only if $K$ is the intersection of a family of finite index subgroups. It is easy to see that a normal subgroup $N \triangleleft G$ is separable if and only if $G / N$ is residually finite. In particular, if $G$ is residually finite and $N \triangleleft G$ is finite, then $G / N$ is also residually finite.

If $H \leqslant G$ has finite index, then $G$ is residually finite if and only if $H$ is. We will also use the following fact: the fundamental group of a finite graph of groups with residually finite vertex groups and finite edge groups is residually finite (see, for instance, [37, II.2.6.12]).

Recall that in a finitely generated group $G$ every finite index subgroup $K \leqslant G$ contains a finite index subgroup $N$ which is characteristic in $G$, e.g., one can take $N=\bigcap_{\alpha \in \operatorname{Aut}(G)} \alpha(K)$. Thus, if $G$ finitely generated and residually finite, then for every $g \in G \backslash\{1\}$ there is a characteristic subgroup $N$ of finite index in $G$ such that $g \notin N$.

\section{Torsion-free hyperbolic groups}

The goal of this section is to give a short proof of the following statement:

Theorem 3.1 Let $G$ be a one-ended hyperbolic group. If $G$ is virtually torsion-free, then $\operatorname{Out}(G)$ is residually finite.

\subsection{Automorphisms with twistors}

Let $H$ be a group. Fix finitely many subgroups $C_{1}, \ldots, C_{s}$ (not necessarily distinct), with $s \geq 1$. We define groups $P M C G(H)$ and $P M C G^{\partial}(H)$ as in Section 4 of [23].

First, $P M C G(H)$ is the subgroup of $\operatorname{Out}(H)$ consisting of all elements $\hat{\alpha}$ represented by automorphisms $\alpha \in \operatorname{Aut}(H)$ acting on each $C_{i}$ as $\tau_{a_{i}}$ for some $a_{i} \in H$.

Let $\operatorname{Aut}^{\partial}(H)$ be the subset of $\operatorname{Aut}(H) \times H^{s}$ given by

$$
\begin{aligned}
\operatorname{Aut}^{\partial}(H)=\left\{\left(\alpha ; a_{1}, \ldots, a_{s}\right) \mid \alpha \in \operatorname{Aut}(H), a_{i} \in H, \alpha(c)=\right. & a_{i} c a_{i}^{-1} \\
& \text { for all } \left.c \in C_{i} \text { and } i=1, \ldots, s\right\} .
\end{aligned}
$$


It is a group, with multiplication defined by

$$
\left(\alpha ; a_{1}, \ldots, a_{s}\right)\left(\alpha^{\prime} ; a_{1}^{\prime}, \ldots, a_{s}^{\prime}\right)=\left(\alpha \circ \alpha^{\prime} ; \alpha\left(a_{1}^{\prime}\right) a_{1}, \ldots, \alpha\left(a_{s}^{\prime}\right) a_{s}\right),
$$

in accordance with the fact that $\alpha \circ \alpha^{\prime}$ acts on $C_{i}$ as conjugation by $\alpha\left(a_{i}^{\prime}\right) a_{i}$.

One easily checks that $I=\left\{\left(\tau_{h} ; h, \ldots, h\right) \in \operatorname{Aut}^{\partial}(H) \mid h \in H\right\}$ is a normal subgroup of $\operatorname{Aut}^{\partial}(H)$. As in [23], we define $P M C G^{\partial}(H)$ to be the quotient of $\operatorname{Aut}^{\partial}(H)$ by $I$. Thus an element of $P M C G^{\partial}(H)$ is represented by $\left(\alpha ; a_{1}, \ldots, a_{s}\right)$, where $\alpha$ is an automorphism of $H$ acting on $C_{i}$ as $\tau_{a_{i}}$, with $a_{i} \in H$. Representatives are defined only up to multiplication by elements of the form $\left(\tau_{h} ; h, \ldots, h\right)$; in particular, for each $i$, there is a unique representative with $a_{i}=1$. Mapping $\left(\alpha ; a_{1}, \ldots, a_{s}\right)$ to $\hat{\alpha}$ defines a projection $\pi: P M C G^{\partial}(H) \rightarrow P M C G(H)$.

As observed in [23, Lemma 4.1] there is a short exact sequence

$$
\{1\} \rightarrow \mathcal{T}_{H} \rightarrow P M C G^{\partial}(H) \stackrel{\pi}{\rightarrow} P M C G(H) \rightarrow\{1\}
$$

whose kernel $\mathcal{T}_{H}$ is the group of twists. It fits in an exact sequence

$$
\{1\} \rightarrow Z(H) \rightarrow \prod_{i=1}^{s} Z_{H}\left(C_{i}\right) \rightarrow \mathcal{T}_{H} \rightarrow\{1\},
$$

where the first map is the diagonal embedding and the second map takes $\left(z_{1}, \ldots, z_{s}\right)$ to the class of $\left(i d_{H} ; z_{1}, \ldots, z_{s}\right)$ in $P M C G^{\partial}(H)$.

If $N \triangleleft H$ is a normal subgroup invariant under $P M C G(H)$, there are natural homomorphisms $P M C G(H) \rightarrow P M C G(H / N)$ and $P M C G^{\partial}(H) \rightarrow P M C G^{\partial}(H / N)$, where the target groups are defined with respect to the images of $C_{1}, \ldots, C_{s}$ in $H / N$.

Lemma 3.2 If $H$ is finitely generated and residually finite, then $P M C G^{\partial}(H)$ is residually finite.

Proof Given any non-trivial $\Phi \in P M C G^{\partial}(H)$, we can construct a characteristic finite index subgroup $N \triangleleft H$ such that $\Phi$ maps non-trivially to the finite group $P M C G^{\partial}(H / N)$. Indeed, let $\left(\alpha ; 1, \ldots, a_{s}\right)$ be the representative of $\Phi$ with $a_{1}=1$. If $\alpha(h) \neq h$ for some $h \in H$, we choose such an $N$ with $h^{-1} \alpha(h) \notin N$. On the other hand, if $a_{i} \neq 1$ for some $i \geq 2$, we choose a characteristic subgroup of finite index $N \triangleleft H$ with $a_{i} \notin N$.

Remark 3.3 There are injective homomorphisms

$$
\operatorname{Aut}^{\partial}(H) \rightarrow H^{s} \rtimes \operatorname{Aut}(H) \text { and } P M C G^{\partial}(H) \rightarrow H^{s-1} \rtimes \operatorname{Aut}(H)
$$

defined by

$$
\left(\alpha ; a_{1}, a_{2}, \ldots, a_{s}\right) \mapsto\left(\left(a_{1}^{-1}, a_{2}^{-1}, \ldots, a_{s}^{-1}\right), \alpha\right) \text { and }\left(\alpha ; 1, a_{2}, \ldots, a_{s}\right) \mapsto\left(\left(a_{2}^{-1}, \ldots, a_{s}^{-1}\right), \alpha\right)
$$

respectively, with $\operatorname{Aut}(H)$ acting on $H^{s}$ and $H^{s-1}$ diagonally. This yields another way of proving Lemma 3.2, using residual finiteness of the semidirect product of a finitely generated residually finite group and a residually finite group.

\subsection{Surfaces}

We now specialize to the case when $H$ is the fundamental group of a compact (possibly nonorientable) surface $\Sigma$ with boundary components $\mathcal{C}_{1}, \ldots, \mathcal{C}_{s}$; we require $s \geq 1$ and $\chi(\Sigma)<$ 0 . We fix a representative $C_{i}$ of $\pi_{1}\left(\mathcal{C}_{i}\right)$ in $G$ and a generator $c_{i}$ of $C_{i}$. Then $P M C G^{\partial}(H)$, as defined above, may be identified with the group of isotopy classes of homeomorphisms of $\Sigma$ equal to the identity on the boundary. In this definition, the isotopy is relative to 
the boundary, so $P M C G^{\partial}(H)$ contains Dehn twists near boundary components. If we do not require isotopies to be relative to the boundary, we get $P M C G(H)$.

There is a central extension

$$
\{1\} \rightarrow \mathcal{T}_{H} \rightarrow \operatorname{PMCG}^{\partial}(H) \rightarrow \operatorname{PMCG}(H) \rightarrow\{1\}
$$

as above, where $\mathcal{T}_{H} \cong \mathbb{Z}^{s}$ is generated by Dehn twists near boundary components of $\Sigma$. The inclusion from $\mathbb{Z}^{s}$ to $P M C G^{\partial}(H)$ may be written algebraically as

$$
\left(n_{1}, \ldots, n_{s}\right) \mapsto\left(i d_{H} ; c_{1}^{n_{1}}, \ldots, c_{s}^{n_{s}}\right) .
$$

Lemma 3.4 Let $n \mathcal{T}_{H} \triangleleft \mathcal{T}_{H}$ be the subgroup generated by the $n$-th powers of the twists. Then $\operatorname{PMCG}^{\partial}(H) / n \mathcal{T}_{H}$ is residually finite for all sufficiently large $n \in \mathbb{N}$.

It is worth noting that residual finiteness does not follow directly from the fact that the group $P M C G^{\partial}(H) / n \mathcal{T}_{H}$ maps onto the residually finite group $P M C G(H)$ with finite kernel.

Proof Let $\Sigma_{n}$ be the closed orbifold obtained by replacing each boundary component of $\Sigma$ by a conical point of order $n$, and let $O_{n}=H /\left\langle\left\langle c_{1}^{n}, \ldots, c_{s}^{n}\right\rangle\right\rangle^{H}$ be its fundamental group.

The Euler characteristic of $\Sigma_{n}$ is $\chi\left(\Sigma_{n}\right)=\chi(\Sigma)+\frac{s}{n}$ (see [36] or [40]). It is negative for $n$ large since $\chi(\Sigma)<0$, so $\Sigma_{n}$ is a hyperbolic orbifold (see [40, Thm. 13.3.6]). It follows that $O_{n}$ embeds into the group of isometries of the hyperbolic plane as a non-elementary subgroup. In particular, $O_{n}$ has trivial center and is residually finite.

Defining $\mathcal{T}_{O_{n}}$ as the kernel of the map $\operatorname{PMCG}^{\partial}\left(O_{n}\right) \rightarrow \operatorname{PMCG}\left(O_{n}\right)$, there is a commutative diagram of short exact sequences

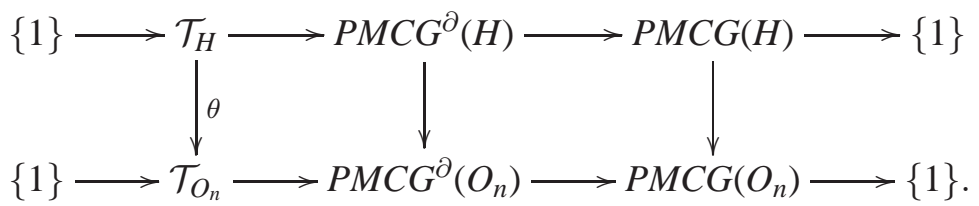

Since $O_{n}$ has trivial center, and the image of $C_{i}$ in $O_{n}$ is equal to its centralizer, $\mathcal{T}_{O_{n}}$ is isomorphic to $(\mathbb{Z} / n \mathbb{Z})^{s}$, so the kernel of the map $\theta$ from $\mathcal{T}_{H}$ to $\mathcal{T}_{O_{n}}$ is precisely $n \mathcal{T}_{H}$ (it is not bigger). The maps from $P M C G^{\partial}(H)$ to $P M C G(H)$ and $P M C G^{\partial}\left(O_{n}\right)$ both factor through $P M C G^{\partial}(H) / n \mathcal{T}_{H}$, and the intersection of their kernels is $\operatorname{ker} \theta=n \mathcal{T}_{H}$. In other words, any non-trivial element of $P M C G^{\partial}(H) / n \mathcal{T}_{H}$ has a non-trivial image in $P M C G(H)$ or in $P M C G^{\partial}\left(O_{n}\right)$. It is well-known that $P M C G(H)$ is residually finite (it is contained in $\operatorname{Out}(H)$, which is residually finite by [15] because $H$ is a finitely generated free group), and $P M C G^{\partial}\left(O_{n}\right)$ is residually finite by Lemma 3.2, so $P M C G^{\partial}(H) / n \mathcal{T}_{H}$ is residually finite.

Remark 3.5 It follows from the classification of non-hyperbolic 2-orbifolds [40] that $n \geq 3$ is always sufficient in Lemma 3.4.

\subsection{An algebraic lemma}

Lemma 3.6 Consider a finite set $V$ and groups $P_{v}, v \in V$, with normal subgroups $T_{v}$ free abelian of finite ranks. Let $P=\prod_{v \in V} P_{v}$ and $T=\prod_{v \in V} T_{v} \leqslant P$ be their direct products. Note that $n T_{v}$ is characteristic in $T_{v}$, hence it is normal in $P_{v}$.

If $P_{v} / n T_{v}$ is residually finite for every $v \in V$ and for all sufficiently large $n \in \mathbb{N}$, then any subgroup $Z \leqslant T$ is closed in the profinite topology of $P$. In particular, if $Z$ is normal in $P$, then $P / Z$ is residually finite. 
Proof Let us first prove the result when $Z$ has finite index $k$ in the free abelian group $T$. It contains $n T$ (with finite index) whenever $k$ divides $n$. For $n$ large, $n T_{v}$ is separable in $P_{v}$ for every $v \in V$ (because $P_{v} / n T_{v}$ is residually finite), so $n T=\prod_{v \in V} n T_{v}$ is separable in $P$, by the properties of direct products. We deduce that $Z$ is closed in $P$, because it is equal to a finite union of cosets modulo $n T$, each of which is separable in $P$ because $n T$ is separable.

The general case follows because $T$ is a free abelian group of finite rank, and therefore every subgroup is the intersection of a collection of finite index subgroups (because the quotient is clearly residually finite).

\subsection{Proof of Theorem 3.1}

First suppose that $G$ is torsion-free. The result is true if $\operatorname{Out}(G)$ is finite, or if $G$ is the fundamental group of a closed surface (in the orientable case this was proved by Grossman [15], and in the non-orientable case by Allenby, Kim and Tang [2]). Otherwise, by Theorem 5.3 of [23], the group $\operatorname{Out}(G)$ is virtually a product $\mathbb{Z}^{q} \times M$, with $M$ a quotient of a finite direct product $\Pi=\prod_{v} P M C G^{\partial}\left(G_{v}\right)$; here $G_{v}$ is a surface group $H$ as in Subsection 3.2 (a QH vertex group of the cyclic JSJ decomposition of $G$ ), and we denote by $\mathcal{T}_{G_{v}} \triangleleft$ $P M C G^{\partial}\left(G_{v}\right)$ the corresponding group of twists. Moreover, the kernel $Z$ of the map from $\Pi$ to $M$ is contained in the free abelian group $\mathcal{T}_{\Pi}=\prod_{v} \mathcal{T}_{G_{v}}$.

Lemma 3.4 implies that $P M C G^{\partial}\left(G_{v}\right) / n \mathcal{T}_{G_{v}}$ is residually finite for all sufficiently large $n$. It follows that $Z$ is separable in $\Pi$ by Lemma 3.6. Thus $M$, and therefore also $\operatorname{Out}(G)$, are residually finite.

Now suppose that $G$ is only virtually torsion-free, and let $N \triangleleft G$ be a torsion-free normal subgroup of finite index. If $G$ is virtually cyclic, $\operatorname{Out}(G)$ is finite (cf. [28, Lemma 6.6]). Otherwise, $N$ has trivial center, so some finite index subgroup of $\operatorname{Out}(G)$ is isomorphic to the quotient of a subgroup of $\operatorname{Out}(N)$ by a finite normal subgroup (see [20, Lemma 5.4] or Lemma 7.15 below). We have shown above that $\operatorname{Out}(N)$ is residually finite, and therefore so is $\operatorname{Out}(G)$.

Remark 3.7 An alternative method to prove Theorem 3.1 could employ Funar's results about residual finiteness of central extensions of mapping class groups [14]. However, writing a complete proof using this approach would still require substantial work, for instance because the surfaces involved may be non-orientable.

\section{Relatively hyperbolic groups and trees}

In this section we recall basic material about relatively hyperbolic groups and trees.

\subsection{Relatively hyperbolic groups}

There are many equivalent definitions of relatively hyperbolic groups in the literature. The definition we give below is due to B. Bowditch [7]; for its equivalence to the other definitions see [22] or [31]. In this paper we will always assume that $G$ and all the groups $P_{i} \in \mathcal{P}$ are finitely generated. (Note that if $G$ is hyperbolic relative to a finite family of finitely generated subgroups then $G$ is itself finitely generated. This follows, for example, from the equivalence of Definition 4.1 with Osin's definition [31, Def. 1.6].) 
Definition 4.1 (Def. 2 of [7]) Consider a group $G$ with a family of subgroups $\mathcal{P}=$ $\left\{P_{1}, \ldots, P_{k}\right\}$. We will say that $G$ is hyperbolic relative to $\mathcal{P}$ if $G$ admits a simplicial action on a connected graph $\mathcal{K}$ such that:

- $\mathcal{K}$ is $\delta$-hyperbolic for some $\delta \geq 0$, and for each $n \in \mathbb{N}$ every edge of $\mathcal{K}$ is contained in finitely many simple circuits of length $n$;

- the edge stabilizers for this action of $G$ on $\mathcal{K}$ are finite, and there are finitely many orbits of edges;

- $\mathcal{P}$ is a set of representatives of conjugacy classes of the infinite vertex stabilizers.

We usually assume that each $P_{i}$ is a proper subgroup of $G$, i.e., $P_{i} \neq G$ (as any $G$ is hyperbolic relative to itself).

A subgroup $H \leqslant G$ is elementary if it is virtually cyclic (possibly finite) or parabolic (contained in a conjugate of some $P_{i}$ ). Any infinite elementary subgroup $H$ is contained in a unique maximal elementary subgroup $\hat{H}$, and $Z_{G}(H) \subset \hat{H}$. We say that $G$ itself is elementary if it is virtually cyclic or equal to some $P_{i}$.

It is well known that, if some $P_{i}$ is virtually cyclic (or, more generally, hyperbolic), then $G$ is hyperbolic relative to the family $\mathcal{P} \backslash\left\{P_{i}\right\}$ - see, for example, [31, Thm. 2.40]. In the context of Corollary 1.1, we may therefore assume that no $P_{i}$ is virtually cyclic. We do not wish to do so in Theorem 1.3, because it may happen that $G$ is one-ended relative to $\mathcal{P}$, but not relative to $\mathcal{P} \backslash\left\{P_{i}\right\}$ with $P_{i}$ infinite and virtually cyclic. For simplicity, however, we assume in most of the paper that no $P_{i}$ is virtually cyclic. The (few) changes necessary to handle the general case are explained in Subsection 7.5.

\subsection{The canonical splitting of a one-ended relatively hyperbolic group}

Assume that $G$ is one-ended, or, more generally, one-ended relative to $\mathcal{P}$ : it does not split over a finite group relative to $\mathcal{P}$ (i.e., with every $P_{i}$ fixing a point in the Bass-Serre tree). Then there is a canonical JSJ tree $T$ over elementary subgroups relative to $\mathcal{P}$ (see [17, Corollary 13.2] and [19]). Canonical means, in particular, that $T$ is invariant under the natural action of $\operatorname{Out}(G ; \mathcal{P})$.

The tree $T$ is equipped with an action of $G$. We denote by $G_{v}$ the stabilizer of a vertex $v$, by $G_{e}$ the stabilizer of an edge $e$ (it is elementary). They are infinite and finitely generated [19]. If $e=v w$, we say that $G_{e}$ is an incident edge stabilizer in $G_{v}$ and $G_{w}$.

We also consider the quotient graph of groups $\Gamma=T / G$. We then denote by $G_{v}$ the group carried by a vertex $v$, by $G_{e}$ the group carried by an edge $e$. If $v$ is an endpoint of $e$, we often identify $G_{e}$ with a subgroup of $G_{v}$, and we say that $G_{e}$ is an incident edge group at $v$.

Being a tree of cylinders (see [17]), $T$ is bipartite, with vertex set $A_{0} \cup A_{1}$. The stabilizer of a vertex $v_{1} \in A_{1}$ is a maximal elementary subgroup (we also say that $v_{1}$ is an elementary vertex). The stabilizer of an edge $\varepsilon=v_{0} v_{1}$ (with $v_{i} \in A_{i}$ ) is a maximal elementary subgroup of $G_{v_{0}}$ (i.e., it is maximal among elementary subgroups contained in $G_{v_{0}}$ ), but $G_{\varepsilon}$ is not necessarily maximal elementary in $G_{v_{1}}$ or in $G$.

Vertices in $A_{0}$ have non-elementary stabilizers. A vertex $v \in A_{0}$ (or its stabilizer $G_{v}$ ) is either rigid or $\mathrm{QH}$ (quadratically hanging). A rigid $G_{v}$ does not split over an elementary subgroup relative to parabolic subgroups and incident edge stabilizers. Through the 
Bestvina-Paulin method and Rips theory, this has strong implications on its automorphisms (see [19]). This will be the key point in the proof of Lemma 5.2.

To describe $\mathrm{QH}$ vertices, it is more convenient to consider a $\mathrm{QH}$ vertex group $G_{v}$ of the graph of groups $\Gamma$. First suppose that $G$ is torsion-free. Then $G_{v}$ may be identified with the fundamental group of a (possibly non-orientable) compact hyperbolic surface $\Sigma_{v}$ whose boundary is non-empty (unless $G_{v}=G$ ).

Moreover, each incident edge group $G_{e}$ is (up to conjugacy) the fundamental group $H_{\mathcal{C}}$ of a boundary component $\mathcal{C}$ of $\Sigma_{v}$. Different edges correspond to different boundary components. Conversely, if no $P_{i}$ is cyclic, the fundamental group $H_{\mathcal{C}}$ of every boundary component $\mathcal{C}$ is an incident edge group. If cyclic $P_{i}$ 's are allowed, it may happen that $H_{\mathcal{C}}$ is not an incident edge group; it is then conjugate to some $P_{i}$.

If torsion is allowed, we only have an exact sequence

$$
\{1\} \rightarrow F \rightarrow G_{v} \stackrel{\xi}{\rightarrow} P \rightarrow\{1\},
$$

where $F$ is a finite group and $P$ is the fundamental group of a compact hyperbolic 2orbifold $\mathcal{O}_{v}$. If $\mathcal{C}$ is a boundary component of $\mathcal{O}_{v}$, its fundamental group $\pi_{1}(\mathcal{C}) \subset P$ is infinite cyclic or infinite dihedral; one defines $H_{\mathcal{C}} \subset G_{v}$ as its full preimage under $\xi$. It is an incident edge group or is conjugate to a virtually cyclic $P_{i}$.

Note that, in all cases, a $\mathrm{QH}$ vertex stabilizer $G_{v}$ of $T$ is virtually free (unless $G_{v}=G$ ), and stabilizers of incident edges are virtually cyclic.

The tree $T$ is relative to $\mathcal{P}$ : every $P_{i}$ fixes a point. If $P_{i}$ is not virtually cyclic, it equals the stabilizer of a vertex $v_{1} \in A_{1}$ or is contained in some $G_{v_{0}}$ with $v_{0}$ rigid (it may happen that $P_{i}=G_{v_{1}} \varsubsetneqq G_{v_{0}}$ ). In particular, the intersection of $P_{i}$ with a $\mathrm{QH}$ vertex group is virtually cyclic. If $P_{i}$ is virtually cyclic and infinite, there is the additional possibility that it is contained in a $\mathrm{QH}$ vertex stabilizer (and conjugate to an $H_{\mathcal{C}}$ as above).

\subsection{The automorphism group of a tree}

Let $T$ be any tree with an action of a finitely generated group $G$. We assume that the action is minimal (there is no proper $G$-invariant subtree), and $T$ is not a point or a line. Let $\operatorname{Out}(G ; T) \subset \operatorname{Out}(G)$ consist of outer automorphisms $\Phi=\hat{\alpha}$ leaving $T$ invariant: in other words, $\Phi$ comes from an automorphism $\alpha \in \operatorname{Aut}(G)$ such that there is an isomorphism $H_{\alpha}: T \rightarrow T$ satisfying $\alpha(g) H_{\alpha}=H_{\alpha} g$ for all $g \in G$. We study $\operatorname{Out}(G ; T)$ as in Sections $2-4$ of $[23]$.

It is more convenient to consider the quotient graph of groups $\Gamma=T / G$. It is finite, and the maps $H_{\alpha}$ induce an action of $\operatorname{Out}(G ; T)$ on $\Gamma$. We denote by $\operatorname{Out}_{0}(G ; T) \leqslant \operatorname{Out}(G ; T)$ the finite index subgroup consisting of automorphisms acting trivially on $\Gamma$.

We denote by $V$ the vertex set of $\Gamma$, by $E$ the set of oriented edges, by $E_{v}$ the set of oriented edges $e$ with origin $o(e)=v$ (incident edges at $v$ ), by $\mathcal{E}$ the set of non-oriented edges. We write $G_{v}$ or $G_{e}$ for the group attached to a vertex or an edge, and we view $G_{e}$ as a subgroup of $G_{v}$ if $e \in E_{v}$ (incident edge group).

For $v \in V$, we define groups $P M C G\left(G_{v}\right) \leqslant \operatorname{Out}\left(G_{v}\right)$ and $P M C G^{\partial}\left(G_{v}\right)$ as in Subsection 3.1, using as $C_{i}$ 's the incident edge groups ( $s$ is the valence of $v$ in $\Gamma$, and there are repetitions if $G_{e}=G_{e^{\prime}}$ with $\left.e \neq e^{\prime}\right)$. We denote by $\pi_{v}: \operatorname{PMCG}^{\partial}\left(G_{v}\right) \rightarrow \operatorname{PMCG}\left(G_{v}\right)$ the natural projection. 
There is a natural map (extension by the identity) $\lambda_{v}: P M C G^{\partial}\left(G_{v}\right) \rightarrow \operatorname{Out}_{0}(G ; T)$ (see $[23$, Section 2.3]). For instance, if $\Gamma$ is an amalgam $G=G_{v} *_{G_{e}} G_{w}$, and $\psi \in P M C G^{\partial}\left(G_{v}\right)$ is represented by $\left(\alpha ; a_{1}\right)$, the image of $\psi$ is represented by the automorphism of $G$ acting as $\alpha$ on $G_{v}$ and as conjugation by $a_{1}$ on $G_{w}$. Elements in the image of $\lambda_{v}$ act as inner automorphisms of $G$ on $G_{w}$ for $w \neq v$. The maps $\lambda_{v}$ have commuting images and fit together in a map

$$
\lambda: \prod_{v \in V} P M C G^{\partial}\left(G_{v}\right) \rightarrow \operatorname{Out}_{0}(G ; T)
$$

There is also a map

$$
\rho=\prod_{v \in V} \rho_{v}: \operatorname{Out}_{0}(G ; T) \rightarrow \prod_{v \in V} \operatorname{Out}\left(G_{v}\right)
$$

recording the action of automorphisms on vertex groups, and the projection

$$
\pi=\prod \pi_{v}: \prod_{v \in V} P M C G^{\partial}\left(G_{v}\right) \rightarrow \prod_{v \in V} P M C G\left(G_{v}\right) \leqslant \prod_{v \in V} \operatorname{Out}\left(G_{v}\right)
$$

factors as $\pi=\rho \circ \lambda$.

We let $\operatorname{Out}_{1}(G ; T) \subset \operatorname{Out}_{0}(G ; T)$ be the image of $\lambda$, and

$$
\rho_{1}: \operatorname{Out}_{1}(G ; T) \rightarrow \prod_{v \in V} \operatorname{PMCG}\left(G_{v}\right)
$$

the restriction of $\rho$. In general $\operatorname{Out}_{1}(G ; T)$ is smaller than $\operatorname{Out}_{0}(G ; T)$ because elements of $\operatorname{Out}_{1}(G ; T)$ are required to map into $\operatorname{PMCG}\left(G_{v}\right)$ for all $v$, and also since ker $\rho$ may fail to be contained in $\operatorname{Out}_{1}(G ; T)$ because of "bitwists" (which will not concern us here, see the proof of Lemma 5.2).

To sum up, we have written $\pi$ as the product of two epimorphisms

$$
\prod_{v \in V} P M C G^{\partial}\left(G_{v}\right) \stackrel{\lambda}{\rightarrow} \operatorname{Out}_{1}(G ; T) \stackrel{\rho_{1}}{\rightarrow} \prod_{v \in V} P M C G\left(G_{v}\right)
$$

We now study the group of twists $\mathcal{T}=\operatorname{ker} \rho_{1}=\operatorname{ker} \rho \cap \operatorname{Out}_{1}(G ; T)$. It is generated by the commuting subgroups $\lambda_{v}\left(\mathcal{T}_{v}\right)$, where $\mathcal{T}_{v}$ is the kernel of the projection $\pi_{v}: \operatorname{PMCG}^{\partial}\left(G_{v}\right) \rightarrow$ $P M C G\left(G_{v}\right)$. As in Subsection 3.1, $\mathcal{T}_{v}$ is the quotient of $\prod_{e \in E_{v}} Z_{G_{v}}\left(G_{e}\right)$ by $Z\left(G_{v}\right)$ (embedded diagonally), which we call a vertex relation. The image of an element $z \in Z_{G_{v}}\left(G_{e}\right)$ in $\operatorname{Out}(G ; T)$ is the twist by $z$ around $e$ near $v$ (note that $z$ does not have to belong to $G_{e}$ ). For instance, in the case of the amalgam considered above, it acts as the identity on $G_{v}$ and as conjugation by $z$ on $G_{w}$.

The group $\mathcal{T}$ is generated by the product $\prod_{e \in E} Z_{G_{o(e)}}\left(G_{e}\right)$. A complete set of relations is given by the vertex relations $Z\left(G_{v}\right)$ (with $Z\left(G_{v}\right)$ embedded diagonally into the factors $\prod Z_{G_{o(e)}}\left(G_{e}\right)$ such that $o(e)=v$ ) and the edge relations $Z\left(G_{e}\right)$ (with $Z\left(G_{e}\right)$ embedded diagonally into the factors $Z_{G_{v}}\left(G_{e}\right)$ and $Z_{G_{w}}\left(G_{\bar{e}}\right)$ if $e$ is an oriented edge $v w$ and $\bar{e}=w v$ ). In the case of an amalgam $G=G_{v} *_{G_{e}} G_{w}$, the edge relation simply says that conjugating both $G_{v}$ and $G_{w}$ by $z \in Z\left(G_{e}\right)$ defines an inner automorphism of $G$.

In other words, $\mathcal{T}$ is the quotient of

$$
\prod_{e \in E} Z_{G_{o(e)}}\left(G_{e}\right)
$$

by the image of

$$
\prod_{v \in V} Z\left(G_{v}\right) \times \prod_{\varepsilon \in \mathcal{E}} Z\left(G_{\varepsilon}\right)
$$


where the products are taken over all oriented edges, all vertices, and all non-oriented edges respectively.

Dividing $\prod_{e \in E} Z_{G_{o(e)}}\left(G_{e}\right)$ by the vertex relations yields $\prod_{v \in V} P M C G^{\partial}\left(G_{v}\right)$. The edge relations generate the kernel of $\lambda: \prod_{v \in V} P M C G^{\partial}\left(G_{v}\right) \rightarrow \operatorname{Out}_{0}(G ; T)$. Note that $\operatorname{ker} \lambda \subset \operatorname{ker} \pi=$ $\prod_{v \in V} \mathcal{T}_{v}$

Example In the case of an amalgam, $\mathcal{T}$ is the image of the map $p: Z_{G_{v}}\left(G_{e}\right) \times Z_{G_{w}}\left(G_{e}\right) \rightarrow$ $\operatorname{Out}(G)$ sending $(a, b)$ to the class of the automorphism acting on $G_{v}$ as conjugation by $b$ and on $G_{w}$ as conjugation by $a$. The kernel of $p$ is generated by the elements $(a, 1)$ with $a \in Z\left(G_{v}\right)$ and $(1, b)$ with $b \in Z\left(G_{w}\right)$ (vertex relations), together with the elements $(c, c)$ with $c \in Z\left(G_{e}\right)$ (edge relations). We have $\mathcal{T}_{v}=Z_{G_{v}}\left(G_{e}\right) / Z\left(G_{v}\right)$ and $\mathcal{T}_{w}=Z_{G_{w}}\left(G_{e}\right) / Z\left(G_{w}\right)$. The kernels of $\lambda: P M C G^{\partial}\left(G_{v}\right) \times P M C G^{\partial}\left(G_{w}\right)$, and of its restriction to $\mathcal{T}_{v} \times \mathcal{T}_{w}$, are generated by $Z\left(G_{e}\right)$.

The following lemma will be used in Section 6 .

Lemma 4.2 Let $W \subset V$. Assume that $G_{v}$ has trivial center if $v \notin W$, and that $\Gamma$ has no edge with both endpoints in $W$. Then the map $\lambda_{W}: \prod_{v \in W} P M C G^{\partial}\left(G_{v}\right) \rightarrow \operatorname{Out}(G ; T)$ is injective.

Proof The kernel of $\lambda_{W}$ is the intersection of ker $\lambda$ with $\prod_{v \in W} \mathcal{T}_{v} \subset \prod_{v \in V} \mathcal{T}_{v}$. If $v \notin W$, the group $G_{v}$ has trivial center, so the product $\prod_{e \in E_{v}} Z\left(G_{e}\right)$, taken over all edges with origin $v$, injects into $\mathcal{T}_{v}$. This implies that, if a product $z=\prod_{\varepsilon \in \mathcal{E}} z_{\varepsilon}$ with $z_{\varepsilon} \in Z\left(G_{\varepsilon}\right)$ maps to $\prod_{v \in W} \mathcal{T}_{v}$, it cannot involve edges having an endpoint outside of $W$. Thus $z$ is trivial since all edges are assumed to have an endpoint not in $W$.

Remark 4.3 If edges with both endpoints in $W$ are allowed, the proof shows that the kernel of $\lambda_{W}$ is generated by the edge relations associated to these edges.

\section{$5 \quad$ Getting rid of the rigids}

Let $G$ be a group hyperbolic relative to a family of finitely generated subgroups $\mathcal{P}=$ $\left\{P_{1}, \ldots, P_{k}\right\}$, and one-ended relative to $\mathcal{P}$. In this section we assume that no $P_{i}$ is virtually cyclic (see Subsection 7.5 for a generalization).

We consider the canonical elementary JSJ tree $T$ relative to $\mathcal{P}$ (see Subsection 4.2). It is invariant under $\operatorname{Out}(G ; \mathcal{P})$, so $\operatorname{Out}(G ; \mathcal{P}) \leqslant \operatorname{Out}(G ; T)$, and bipartite: each edge joins a vertex with elementary stabilizer to a vertex with non-elementary (rigid or $\mathrm{QH}$ ) stabilizer. In particular, $T$ cannot be a line; we assume that it is not a point.

As above, we consider the quotient graph of groups $\Gamma=T / G$, with vertex set $V$. Just like those of $T$, vertices of $\Gamma$ (and their groups) may be elementary, rigid, or QH. We partition $V$ as $V_{E} \cup V_{R} \cup V_{Q H}$ accordingly; each edge has exactly one endpoint in $V_{E}$.

Lemma 5.1 $\mathcal{T}$ is generated by the groups $\lambda_{w}\left(\mathcal{T}_{w}\right)$ with $w \in V_{E}$ : twists near vertices in $V_{E}$ generate the whole group of twists of $T$.

Proof If $e=v w$ is any edge with $v \in V_{Q H} \cup V_{R}$ (and therefore $w \in V_{E}$ ), then $G_{e}$ is a maximal elementary subgroup of $G_{v}$, so $Z_{G_{v}}\left(G_{e}\right)=Z\left(G_{e}\right)$ since $Z_{G_{v}}\left(G_{e}\right) \subset G_{e}$. We can then use edge relations to view twists around $e$ near $v$ as twists near $w$. 
Lemma 5.2 Let $\operatorname{Out}^{r}(G)$ be the image of the restriction

$$
\lambda_{E, Q H}: \prod_{v \in V_{E} \cup V_{Q H}} P M C G^{\partial}\left(G_{v}\right) \rightarrow \operatorname{Out}(G)
$$

of $\lambda$ (see Subsection 4.3). Then $\operatorname{Out}^{r}(G)$ is contained in $\operatorname{Out}(G ; \mathcal{P})$ with finite index.

Proof We first prove $\operatorname{Out}^{r}(G) \subset \operatorname{Out}(G ; \mathcal{P})$. Recall that $P_{i}$ is assumed not to be virtually cyclic, so (up to conjugacy) it is equal to an elementary vertex group or is contained in a rigid vertex group. In either case elements of $\operatorname{Out}^{r}(G)$ map $P_{i}$ to a conjugate (trivially if $P_{i}$ is contained in a rigid group). In fact, $\operatorname{Out}^{r}(G)$ is contained in $\operatorname{Out}_{0}(G ; \mathcal{P})=\operatorname{Out}(G ; \mathcal{P}) \cap$ $\operatorname{Out}_{0}(G ; T)$, a finite index subgroup of $\operatorname{Out}(G ; \mathcal{P})$. We show that $\operatorname{Out}^{r}(G)$ has finite index in $\mathrm{Out}_{0}(G ; \mathcal{P})$.

Recall the maps $\rho_{v}: \operatorname{Out}_{0}(G ; T) \rightarrow \operatorname{Out}\left(G_{v}\right)$, and consider their restrictions to the subgroup $\operatorname{Out}_{0}(G ; \mathcal{P})$. It is shown in Proposition 4.1 of [19] that the image of such a restriction is finite if $v \in V_{R}$ (this is a key property of rigid vertices), contains $P M C G\left(G_{v}\right)$ with finite index if $v \notin V_{R}$ (note that $\operatorname{PMCG}\left(G_{v}\right)$ is $\operatorname{Out}\left(G_{v} ; \operatorname{Inc}_{v}^{(t)}\right)$ in [19]; the assumption that no $P_{i}$ is virtually cyclic is used to ensure $\mathcal{B}_{v}=\operatorname{Inc}_{v}$ ).

Now consider the homomorphism $\rho: \operatorname{Out}_{0}(G ; T) \rightarrow \prod_{v \in V} \operatorname{Out}\left(G_{v}\right)$. The image of $\operatorname{Out}^{r}(G)$ is $\prod_{v \in V_{E} \cup V_{Q H}} P M C G\left(G_{v}\right)$, so it has finite index in the image of $\operatorname{Out}_{0}(G ; \mathcal{P})$. We complete the proof by showing that $\operatorname{Out}^{r}(G)$ contains $\operatorname{ker} \rho$.

It is pointed out in Subsection 3.3 of [19] that $\operatorname{ker} \rho$ is equal to the group of twists $\mathcal{T}$ (because, if $v \notin V_{E}$, then incident edge groups are equal to their normalizer in $G_{v}$ ). By Lemma 5.1, $\mathcal{T}$ is generated by twists near vertices in $V_{E}$. These belong to the image of $\lambda_{E, Q H}$, so $\mathcal{T} \subset \operatorname{Out}^{r}(G)$.

We shall now change the graph of groups $\Gamma$ into a new graph of groups $\Gamma^{\prime}$. We do not change the underlying graph, or edge groups, or vertex groups $G_{v}$ for $v \in V_{E} \cup V_{Q H}$, but for $v \in V_{R}$ we replace $G_{v}$ by a group $G_{v}^{\prime}$ defined as follows.

If $e \in E_{v}$ is an incident edge, $G_{e}$ is a maximal elementary subgroup of $G_{v}$, in particular it contains the finite group $Z\left(G_{v}\right)$. Consider the groups $G_{e}$, for $e \in E_{v}$, as well as $\mathbb{Z} \times Z\left(G_{v}\right)$. All these groups contain $Z\left(G_{v}\right)$, and we define $G_{v}^{\prime}$ as their free amalgam over $Z\left(G_{v}\right)$, i.e., $G_{v}^{\prime}$ is obtained from the free product $\left(*_{e \in E_{v}} G_{e}\right) *\left(\mathbb{Z} \times Z\left(G_{v}\right)\right)$ by identifying all copies of $Z\left(G_{v}\right)$.

The inclusion from $G_{e}$ into the new vertex group $G_{v}^{\prime}$ is the obvious one. Note that $G_{v}^{\prime}$ is not one-ended relative to incident edge groups (because of the factor $\mathbb{Z} \times Z\left(G_{v}\right)$ ), and is residually finite if the $P_{i}$ 's are (as an amalgam of residually finite groups over a finite subgroup). We denote by $G^{\prime}$ the fundamental group of $\Gamma^{\prime}$.

Lemma 5.3 The finite index subgroup $\operatorname{Out}^{r}(G) \subset \operatorname{Out}(G ; \mathcal{P})$ is isomorphic to a subgroup of $\operatorname{Out}\left(G^{\prime}\right)$.

Proof Since nothing changes near vertices in $V_{E} \cup V_{Q H}$, we still have a map

$$
\lambda_{E, Q H}^{\prime}: \prod_{v \in V_{E} \cup V_{Q H}} \operatorname{PMCG}^{\partial}\left(G_{v}\right) \rightarrow \operatorname{Out}\left(G^{\prime}\right) .
$$

It suffices to show $\operatorname{ker} \lambda_{E, Q H}^{\prime}=\operatorname{ker} \lambda_{E, Q H}$. Recall that the kernel of $\lambda_{E, Q H}$ is the same as the kernel of its restriction to $\prod_{v \in V_{E} \cup V_{Q H}} \mathcal{T}_{v}$, and similarly for $\lambda_{E, Q H}^{\prime}$. For $v \in V_{R}$, the group $G_{v}^{\prime}$ was defined so that the groups $Z\left(G_{v}\right)$ and $Z_{G_{v}}\left(G_{e}\right)$ do not change, so $\mathcal{T}_{v}$ does not change, and neither does the map from $\prod_{v \in V} \mathcal{T}_{v}$ to $\operatorname{Out}(G)$. The result follows. 


\section{Torsion-free relatively hyperbolic groups}

This section is devoted to a proof of Theorem 1.3 in the torsion-free case.

Theorem 6.1 Let $G$ be a torsion-free group hyperbolic relative to a family of proper finitely generated residually finite subgroups $\mathcal{P}=\left\{P_{1}, \ldots, P_{k}\right\}$, none of which is cyclic. If $G$ is one-ended relative to $\mathcal{P}$, then $\operatorname{Out}(G ; \mathcal{P})$ is residually finite.

See Remark 6.2 for the case when some of the $P_{i}$ 's are allowed to be cyclic.

Proof As in the previous section, let $\Gamma$ be the canonical JSJ decomposition of $G$. First suppose that $\Gamma$ is trivial (a single vertex $v$ ). If $v$ is $\operatorname{rigid}$, then $\operatorname{Out}(G ; \mathcal{P}$ ) is finite (see [19]). If $v$ is QH, then $G$ is a closed surface group and $\operatorname{Out}(G)$ is residually finite by $[15,2]$. The case $v \in V_{E}$ cannot occur since $P_{i} \neq G$. From now on, we suppose that $\Gamma$ is nontrivial.

By Lemma 5.2, it is enough to show that $\operatorname{Out}^{r}(G)$ is residually finite. Given a nontrivial $\Phi \in \operatorname{Out}^{r}(G)$, we want to map $\operatorname{Out}^{r}(G)$ to a finite group without killing $\Phi$. Note that it is enough to map $\operatorname{Out}^{r}(G)$ to a residually finite group without killing $\Phi$.

Using Lemma 5.3, we view $\operatorname{Out}^{r}(G)$ as a group of automorphisms of $G^{\prime}$. To simplify notation, we will not write the superscripts ' unless necessary.

Recall the epimorphisms

$$
\prod_{v \in V_{E} \cup V_{Q H}} P M C G^{\partial}\left(G_{v}\right) \rightarrow \operatorname{Out}^{r}(G) \rightarrow \prod_{v \in V_{E} \cup V_{Q H}} \operatorname{PMCG}\left(G_{v}\right)
$$

induced by $\lambda_{E, Q H}$ and $\prod_{v \in V_{E} \cup V_{Q H}} \rho_{v}$. Write $\Phi$ as the image, under $\lambda_{E, Q H}$, of a tuple $\left(\Phi_{v}\right) \in \prod_{v \in V_{E} \cup V_{Q H}} P M C G^{\partial}\left(G_{v}\right)$. If $v \in V_{Q H}$, the group $P M C G\left(G_{v}\right)$ is residually finite since $\operatorname{PMCG}\left(G_{v}\right) \subset \operatorname{Out}\left(G_{v}\right)$ with $G_{v}$ free of finite rank, so we may assume that $\Phi$ maps trivially to $P M C G\left(G_{v}\right)$ for $v \in V_{Q H}$. This means that $\Phi_{v} \in \mathcal{T}_{v} \subset P M C G^{\partial}\left(G_{v}\right)$ for $v \in V_{Q H}$. By Lemma 5.1, we may use edge relations to find a representative of $\Phi$ with $\Phi_{v}=1$ for $v \in V_{Q H}$. We fix such a representative, and we choose $u \in V_{E}$ with $\Phi_{u} \neq 1$.

Next we fix a characteristic finite index subgroup $N_{v} \triangleleft G_{v}$ for each $v \in V_{E}$, and we denote $H_{v}=G_{v} / N_{v}$. Since $G_{v}$ is assumed to be residually finite (it is cyclic or conjugate to a $P_{i}$ ), we may also require that, if $e=v w$ is an edge with $w \in V_{Q H}$, then the image of $G_{e} \cong \mathbb{Z}$ in $H_{v}$ has order $n_{e} \geq 4$. As in the proof of Lemma 3.2, we also require that $\Phi_{u}$ maps to a non-trivial element under the natural homomorphism from $\operatorname{PMCG}^{\partial}\left(G_{u}\right)$ to $\operatorname{PMCG}^{\partial}\left(H_{u}\right)$.

We now construct a new graph of groups $\Gamma^{\prime \prime}$, with the same underlying graph as $\Gamma$ and $\Gamma^{\prime}$, with vertex groups $G_{v}^{\prime \prime}=H_{v}$ at vertices $v \in V_{E}$. We describe the edge groups, and the other vertex groups. The inclusions from edge groups to vertex groups will be the obvious ones.

Given an edge $e=v w$ with $v \in V_{E}$, its group $G_{e}^{\prime \prime}$ in $\Gamma^{\prime \prime}$ is the image of $G_{e}$ in $H_{v}$, a cyclic group of order $n_{e} \geq 4$.

If $v \in V_{R}$, its group in $\Gamma^{\prime}$ is the free product $\left(* G_{e}\right) * \mathbb{Z}$, with the first product taken over all $e \in E_{v}$. We define its new group as $G_{v}^{\prime \prime}=\left(* G_{e}^{\prime \prime}\right) * \mathbb{Z}$.

If $v \in V_{Q H}$, its group in $\Gamma$ and $\Gamma^{\prime}$ is a surface group $\pi_{1}\left(\Sigma_{v}\right)$, with boundary components of $\Sigma_{v}$ corresponding to incident edges (see Subsection 4.2). We define $G_{v}^{\prime \prime}$ as the fundamental group of the closed orbifold obtained from $\Sigma_{v}$ by replacing the boundary component associated to an edge $e$ by a conical point of order $n_{e}$. The assumption $n_{e} \geq 4$ ensures 
that the orbifold is hyperbolic (see [40], 13.3.6), so $G_{v}^{\prime \prime}$ is a non-elementary subgroup of $\operatorname{Isom}\left(\mathbb{H}^{2}\right)$. In particular, $G_{v}^{\prime \prime}$ has trivial center and is residually finite.

The fundamental group $G^{\prime \prime}$ of $\Gamma^{\prime \prime}$ is a quotient of $G^{\prime}$. It is residually finite, as the fundamental group of a graph of groups with residually finite vertex groups and finite edge groups. We show that the splitting $\Gamma^{\prime \prime}$ of $G^{\prime \prime}$ is nontrivial.

If $v \notin V_{E}$, the group $G_{v}^{\prime \prime}$ is infinite (because of the factor $\mathbb{Z}$ in the rigid case), so triviality would imply that $u$ has valence 1 and the incident edge group in $\Gamma^{\prime \prime}$ is equal to $G_{u}^{\prime \prime}$. This is impossible because $P M C G^{\partial}\left(G_{u}^{\prime \prime}\right)$ is non-trivial (it contains the image of $\Phi_{u}$, which is assumed to be non-trivial).

Since $\Gamma^{\prime \prime}$ is a splitting over finite groups, $G^{\prime \prime}$ has infinitely many ends. By [28, Thm. 1.5], $\operatorname{Out}\left(G^{\prime \prime}\right)$ is residually finite. We conclude the proof by constructing a map from $\operatorname{Out}^{r}(G)$ to $\operatorname{Out}\left(G^{\prime \prime}\right)$ mapping $\Phi$ non-trivially.

Let $v \in V_{E} \cup V_{Q H}$. Since the kernel of the map $G_{v} \rightarrow G_{v}^{\prime \prime}$ is invariant under $\operatorname{PMCG}\left(G_{v}\right)$, there is an induced map from $P M C G^{\partial}\left(G_{v}\right)$ to $P M C G^{\partial}\left(G_{v}^{\prime \prime}\right)$. Similarly, the kernel of the projection from $G^{\prime}$ to $G^{\prime \prime}$ is invariant under the image of $\operatorname{Out}^{r}(G)$ in $\operatorname{Out}\left(G^{\prime}\right)$ (see Lemma $5.3)$, so there is an induced map from $\operatorname{Out}^{r}(G)$ to $\operatorname{Out}\left(G^{\prime \prime}\right)$. These maps fit in a commutative diagram

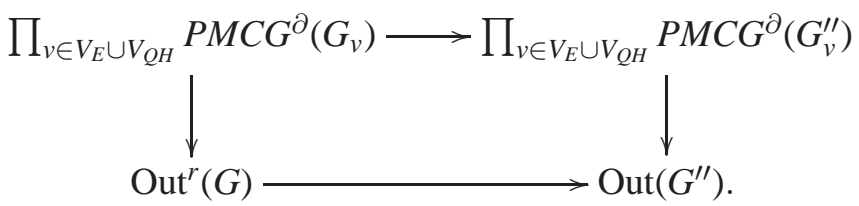

Since $\Phi_{v}=1$ for $v \in V_{Q H}$, and $\Phi_{u}$ maps non-trivially to $P M C G^{\partial}\left(H_{u}\right)$, Lemma 4.2 (applied in $\Gamma^{\prime \prime}$ with $\left.W=V_{E}\right)$ implies that $\Phi$ maps non-trivially to $\operatorname{Out}\left(G^{\prime \prime}\right)$.

The main difficulty in extending this proof to groups with torsion lies in defining $G_{v}^{\prime \prime}$ for $v \in V_{Q H}$ when $G_{v}$ contains a nontrivial finite normal subgroup $F$. This may be done using small cancellation techniques, but we prefer to give a different proof (using Grossman's method and Dehn fillings) in the general case.

Remark 6.2 Theorem 6.1 holds if the $P_{i}$ 's are allowed to be cyclic ( recall that an infinitely-ended $G$ may become one-ended relative to $\mathcal{P}$ if cyclic $P_{i}$ 's are added). There is a technical complication due to the fact that, if $G_{v}=\pi_{1}\left(\Sigma_{v}\right)$ is QH, there may exist boundary components of $\Sigma_{v}$ whose fundamental group equals some $P_{i}$ (up to conjugacy) but is not an incident edge group. Because of this, one must change the definition of $P M C G^{\partial}\left(G_{v}\right)$ and $P M C G\left(G_{v}\right)$ slightly. See Subsection 7.5 for details.

\section{Groups with torsion}

The goal of this section is to prove Theorem 1.3 (Subsections 7.4 and 7.5) and Corollary 1.1 (Subsection 7.6).

\subsection{Finitary fillings of relatively hyperbolic groups}

Definition 7.1 Let $\mathcal{I}$ be a property of groups, let $G$ be a group, and let $\left\{H_{\lambda}\right\}_{\lambda \in \Lambda}$ be a non-empty collection of subgroups of $G$. We will say that most finitary fillings of $G$ with respect to $\left\{H_{\lambda}\right\}_{\lambda \in \Lambda}$ have property $\mathcal{I}$ provided there is a finite subset $S \subset G \backslash\{1\}$ such that, for any family of finite index normal subgroups $N_{\lambda} \triangleleft H_{\lambda}$ with $N_{\lambda} \cap S=\emptyset$ for all $\lambda \in \Lambda$, the following two conditions hold: 
- for each $\lambda \in \Lambda$ one has $N \cap H_{\lambda}=N_{\lambda}$, where $N:=\left\langle\left\langle N_{\lambda} \mid \lambda \in \Lambda\right\rangle\right\rangle^{G}$;

- the quotient $G / N$ satisfies $\mathcal{I}$.

Any quotient $G / N$ as above will be called a finitary filling of $G$ with respect to $\left\{H_{\lambda}\right\}_{\lambda \in \Lambda}$. The finite subset $S$ will be called the obstacle subset.

In this work we will mainly be concerned with the case when $\mathcal{I}$ is the property of being residually finite, or conjugacy separable.

Remark 7.2 If $G=A *_{C} B$, where $C$ is finite and $A, B$ are residually finite, then most finitary fillings of $G$ with respect to any of the families $\{A\},\{B\}$ or $\{A, B\}$ are residually finite (it suffices to take $S:=C \backslash\{1\}$ and use the universal property of amalgamated free products; $G / N$ will be an amalgam of residually finite groups over $C$ ). More generally, if $G$ is the fundamental group of a finite graph of groups with finite edge groups and residually finite vertex groups, then most finitary fillings of $G$ with respect to any collection of vertex groups will be residually finite.

In this subsection, and the next, we will consider a graph of groups $\Gamma$, with fundamental group $G$, satisfying the following assumption (this will be applied to the graph $\Gamma^{\prime}$ constructed in Section 5).

Assumption 7.3 $\Gamma$ is a connected finite bipartite graph with vertex set $V=V_{1} \sqcup V_{2}$ (so every vertex from $V_{1}$ is only adjacent to vertices from $V_{2}$ and vice-versa), and $\Gamma$ is not a point. Moreover, the following properties hold:

(1) if $u \in V_{1}$ then the group $G_{u}$ is residually finite;

(2) if $v \in V_{2}$ then most finitary fillings of $G_{v}$ with respect to $\left\{G_{e_{1}}, \ldots, G_{e_{s}}\right\}$ are residually finite, with $e_{1}, \ldots, e_{s}$ the collection of all oriented edges of $\Gamma$ starting at $v$, and $G_{e_{1}}, \ldots, G_{e_{s}}$ the corresponding edge groups;

(3) for every $u \in V_{1}$, the group $G_{u}$ is a proper finitely generated subgroup of $G=\pi_{1}(\Gamma)$, and $G$ is hyperbolic relative to the family $\left\{G_{u} \mid u \in V_{1}\right\}$; in particular, $G$ is finitely generated.

The main technical tool for our approach is the theory of Dehn fillings in relatively hyperbolic groups, developed by Osin - see [29, Thm. 1.1] (in the torsion-free case this was also done independently by Groves and Manning - see [16, Thm. 7.2]):

Theorem 7.4 Suppose that a group $G$ is hyperbolic relative to a family of subgroups $\left\{H_{\lambda}\right\}_{\lambda \in \Lambda}$. Then there exists a finite subset $S \subset G \backslash\{1\}$ with the following property. If $\left\{N_{\lambda}\right\}_{\lambda \in \Lambda}$ is any collection of subgroups such that $N_{\lambda} \triangleleft H_{\lambda}$ and $N_{\lambda} \cap S=\emptyset$ for all $\lambda \in \Lambda$, then:

1) for each $\lambda \in \Lambda$ one has $H_{\lambda} \cap N=N_{\lambda}$, where $N:=\left\langle\left\langle N_{\lambda} \mid \lambda \in \Lambda\right\rangle\right\rangle^{G}$;

2) the quotient group $G / N$ is hyperbolic relative to the collection $\left\{H_{\lambda} / N_{\lambda}\right\}_{\lambda \in \Lambda}$.

Moreover, for any finite subset $M \subset G$, there exists a finite subset $S(M) \subset G \backslash\{1\}$, such that the restriction of the natural homomorphism $G \rightarrow G / N$ to $M$ is injective whenever $N_{\lambda} \cap S(M)=\emptyset$ for all $\lambda \in \Lambda$.

Suppose that $\Delta$ is any graph of groups with fundamental group $G$, and we are given normal subgroups $N_{v} \triangleleft G_{v}$ for each vertex $v$. Assume furthermore that $N_{v} \cap G_{e}=N_{w} \cap G_{e}$ whenever $e=v w$ is an edge of $\Delta$ (as usual, we view $G_{e}$ as a subgroup of both $G_{v}$ and $G_{w}$; 
to be precise, we want the preimages of $N_{v}$ and $N_{w}$, under the embeddings of $G_{e}$ into $G_{v}$ and $G_{w}$ respectively, to coincide).

Then we can construct a "quotient graph of groups" $\bar{\Delta}$ as follows: the underlying graph is the same as in $\Delta$, the vertex group at a vertex $v$ is $G_{v} / N_{v}$, the group carried by $e=v w$ is $G_{e} /\left(N_{v} \cap G_{e}\right)$, and the inclusions are the obvious ones. The fundamental group of $\bar{\Delta}$ is isomorphic to $G /\left\langle\left\langle\cup_{v} N_{v}\right\rangle\right)^{G}$.

Now suppose that $\Gamma$ is as in Assumption 7.3. For each $v \in V_{2}$, there is an obstacle set $S_{v} \subset G_{v} \backslash\{1\}$, and we define $S:=\bigcup_{v \in V_{2}} S_{v}$.

Lemma 7.5 Consider an arbitrary family of subgroups $\left\{N_{u}\right\}_{u \in V_{1}}$ such that $N_{u} \triangleleft G_{u}$, $\left|G_{u}: N_{u}\right|<\infty$ and $N_{u} \cap S=\emptyset$ for every $u \in V_{1}$. The group $\bar{G}:=G /\left\langle\left\langle\cup_{u \in V_{1}} N_{u}\right\rangle\right\rangle^{G}$ is the fundamental group of a quotient graph of groups $\bar{\Gamma}$ in which every $u \in V_{1}$ carries $G_{u} / N_{u}$, and every $v \in V_{2}$ carries a residually finite group. In particular, $\bar{G}$ is residually finite.

Proof For every edge $e=u v$ of $\Gamma$, with $u \in V_{1}$ and $v \in V_{2}$, we define a finite index normal subgroup $L_{e} \triangleleft G_{e}$ by $L_{e}:=G_{e} \cap N_{u}$ (as above, we view $G_{e}$ as a subgroup of both $G_{u}$ and $\left.G_{v}\right)$. Now, for each vertex $v \in V_{2}$ we let $M_{v}:=\left\langle\left\langle L_{e_{1}} \cup \cdots \cup L_{e_{s}}\right\rangle\right\rangle^{G_{v}} \triangleleft G_{v}$, where $e_{1}, \ldots, e_{s}$ are the edges of $\Gamma$ starting at $v$. Observe that $L_{e_{j}} \cap S_{v}=\emptyset$ for $j=1, \ldots, s$ by construction, hence $M_{v} \cap G_{e_{j}}=L_{e_{j}}$ by Definition 7.1.

This shows that $\bar{G}$ is represented by a quotient graph of groups $\bar{\Gamma}$. The group carried by $u \in V_{1}$ is $G_{u} / N_{u}$, a finite group; in particular, edge groups are finite. The group carried by $v \in V_{2}$ is $G_{v} / M_{v}$, which is residually finite by Assumption 7.3. Thus $\bar{G}$ is residually finite.

Remark 7.6 Since every $G_{u}$, with $u \in V_{1}$, is residually finite, a family of normal subgroups $\left\{N_{u}\right\}_{u \in V_{1}}$ as in Lemma 7.5 exists. We may even require $N_{u} \cap S^{\prime}=\emptyset$ if $S^{\prime}$ is any given finite subset of $G \backslash\{1\}$.

Remark 7.7 Lemma 7.5 only requires the second condition of Assumption 7.3.

Proposition 7.8 Let $G$ be the fundamental group of a graph of groups $\Gamma$ as in Assumption 7.3. Then $G$ is residually finite.

Proof Take any element $x \in G \backslash\{1\}$ and let $M:=\{1, x\} \subset G$. Since each $G_{u}, u \in V_{1}$, is residually finite, one can find sufficiently small finite index subgroups $N_{u} \triangleleft G_{u}$ as in Lemma 7.5. We can also assume that each $N_{u}$ is disjoint from the set $S(M)$ provided by Theorem 7.4 (applied to $G$ relative to the $G_{u}$ 's). If $N:=\left\langle\left\langle N_{u} \mid u \in V_{1}\right\rangle\right\rangle^{G}$, then $\bar{G}=G / N$ is residually finite by Lemma 7.5, and the image of $x$ under the map $\kappa: G \rightarrow \bar{G}$ is non-trivial by the final claim of Theorem 7.4. Composing $\kappa$ with a map from $\bar{G}$ to a finite group that does not kill $\kappa(x)$, we get a finite quotient of $G$ in which the image of $x$ is non-trivial. This shows residual finiteness of $G$.

The above proposition is true even without the hypothesis that vertex groups from $V_{1}$ are finitely generated. 


\subsection{Using Grossman's method}

Let $G$ be hyperbolic relative to a family of proper finitely generated subgroups $\mathcal{P}=$ $\left\{P_{1}, \ldots, P_{k}\right\}$.

Recall that an element $g \in G$ is called loxodromic if $g$ has infinite order and is not conjugate to an element of $P_{i}$ for any $i$. Two elements $g, h \in G$ are said to be commensurable in $G$ if there are $f \in G$ and $m, n \in \mathbb{Z} \backslash\{0\}$ such that $h^{n}=f g^{m} f^{-1}$ (we use the terminology of [28], where conjugate elements are considered commensurable). An automorphism $\alpha \in \operatorname{Aut}(G)$ is called commensurating if $\alpha(g)$ is commensurable with $g$ for every $g \in G$.

It is known that $G$ contains a unique maximal finite normal subgroup denoted $E(G)$ (see [28, Cor. 2.6]); this subgroup contains the center of $G$ if $G$ is not virtually cyclic.

Lemma 7.9 Assume that $G$ is not virtually cyclic.

(1) If $E(G)=\{1\}$, then every commensurating automorphism of $G$ is inner.

(2) Suppose that $\alpha \in \operatorname{Aut}(G)$ is not a commensurating automorphism. Then there exists a loxodromic element $g \in G$ such that $\alpha(g)$ is also loxodromic and $\alpha(g)$ is not commensurable with $g$ in $G$.

Proof The statement (1) is proved in [28, Cor. 1.4].

Suppose that $\alpha \in \operatorname{Aut}(G)$ is not commensurating. By [28, Cor. 5.3] there exists a loxodromic element $g_{0} \in G$ such that $\alpha\left(g_{0}\right)$ is not commensurable with $g_{0}$ in $G$. The statement (2) now follows after applying [28, Lemma 4.8].

Lemma 7.10 ([28, Lemma 7.1]) Assume that $G$ is hyperbolic relative to $\left\{P_{1}, \ldots, P_{k}\right\}$, with $k \geq 1$, and $g, h \in G$ are two non-commensurable loxodromic elements. Then $g$ and $h$ are loxodromic and non-commensurable in most finitary fillings of $G$ with respect to $\left\{P_{1}, \ldots, P_{k}\right\}$.

Recall that $G$ is conjugacy separable if, given any non-conjugate elements $g, h \in G$, there exists a homomorphism $\varphi$ from $G$ to a finite group such that $\varphi(g)$ and $\varphi(h)$ are not conjugate. Note that this is evidently stronger than residual finiteness of $G$.

Proposition 7.11 Let $G$ be the fundamental group of a graph of groups $\Gamma$ as in Assumption 7.3. Suppose that $E(G)=\{1\}$, and for each $v \in V_{2}$ most finitary fillings of $G_{v}$ with respect to $\left\{G_{e_{1}}, \ldots, G_{e_{s}}\right\}$ (where $e_{1}, \ldots, e_{s}$ is the list of edges of $\Gamma$ starting at $v$ ) are conjugacy separable. Then $\operatorname{Out}(G)$ is residually finite.

The proof uses Grossman's method, which is based on the following fact:

Lemma 7.12 Given a finitely generated group $G$ and $\alpha \in \operatorname{Aut}(G)$, suppose that there is a homomorphism $\psi: G \rightarrow K$ with $K$ finite, and $g \in G$, such that $\psi(g)$ is not conjugate to $\psi(\alpha(g))$ in $K$. Then there is a homomorphism $\hat{\theta}: \operatorname{Out}(G) \rightarrow L$ with $L$ finite such that $\hat{\theta}(\hat{\alpha}) \neq 1$ in $L$, where $\hat{\alpha}$ is the image of $\alpha$ in $\operatorname{Out}(G)$.

Proof Since $G$ is finitely generated, there exists a characteristic finite index subgroup $N \triangleleft G$ such that $N \leq \operatorname{ker} \psi$. Let $\varphi: G \rightarrow G / N$ be the canonical epimorphism. Then $\psi$ factors through $\varphi$, hence $\varphi(g)$ is not conjugate to $\varphi(\alpha(g))$ in $G / N$. Observe that, as $N$ is characteristic in $G$, there are induced homomorphisms $\theta: \operatorname{Aut}(G) \rightarrow \operatorname{Aut}(G / N)$ and $\hat{\theta}: \operatorname{Out}(G) \rightarrow L:=\operatorname{Out}(G / N)$. Since $\varphi(g)$ is not conjugate to $\varphi(\alpha(g))$ in $G / N$, the automorphism $\theta(\alpha)$ is not inner and $\hat{\theta}(\hat{\alpha}) \neq 1$. 
Proof of Proposition 7.11 We may assume that $G$ is not virtually cyclic, since Out $(G)$ is finite if it is (see [28, Lemma 6.6]). Applying Lemma 7.5 and Remark 7.6, we find a quotient graph of groups $\bar{\Gamma}$ where vertices in $V_{1}$ carry finite groups and vertices in $V_{2}$ carry residually finite groups. We write $\bar{G}=\pi_{1}(\bar{\Gamma})$, and we let $\kappa: G \rightarrow \bar{G}$ be the projection.

We shall now enlarge $S$ to ensure that $\bar{\Gamma}$ possesses additional properties. First, we may assume that vertices in $V_{2}$ carry a conjugacy separable group. By [12], $\bar{G}$ is then conjugacy separable, as the fundamental group of a finite graph of groups with conjugacy separable vertex groups and finite edge groups.

Now consider any $\hat{\alpha} \in \operatorname{Out}(G) \backslash\{1\}$, represented by $\alpha \in \operatorname{Aut}(G) \backslash \operatorname{Inn}(G)$. Since $E(G)=\{1\}$, by Lemma 7.9 there exists a loxodromic element $g \in G$ such that $\alpha(g)$ is a loxodromic element not commensurable with $g$ in $G$. By Lemma 7.10 (applied to $G$ relative to the $G_{u}$ 's), we can enlarge the obstacle set $S$ to assume that the elements $\kappa(g)$ and $\kappa(\alpha(g))$ are non-commensurable in $\bar{G}$.

Since $\bar{G}$ is conjugacy separable, there exists a finite group $K$ and a homomorphism $\eta$ : $\bar{G} \rightarrow K$ such that $\eta(\kappa(g))$ is not conjugate to $\eta(\kappa(\alpha(g)))$ in $K$. Thus, setting $\psi:=\eta \circ \kappa$ : $G \rightarrow K$, we can apply Lemma 7.12 to find a finite quotient of $\operatorname{Out}(G)$ separating $\hat{\alpha}$ from the identity.

\subsection{Quadratically hanging groups}

In the next subsection, we will apply Propositions 7.8 and 7.11 to the canonical JSJ decomposition. In order to do this, we need to study finitary fillings of QH vertex groups. We denote such a group by $O$.

Recall from Subsection 4.2 that $O$ is an extension $\{1\} \rightarrow F \rightarrow O \stackrel{\xi}{\rightarrow} P \rightarrow\{1\}$, where $F$ is a finite group and $P$ is the fundamental group of a hyperbolic 2-orbifold $\mathcal{O}$ with boundary. Consider full preimages $C_{i}=\xi^{-1}\left(B_{i}\right)$ of a set of representatives $B_{1}, \ldots, B_{s}$ of fundamental groups of components of the boundary of $\mathcal{O}$.

The goal of this subsection is the following statement:

Proposition 7.13 Most finitary fillings of $O$ with respect to $\mathcal{H}=\left\{C_{1}, \ldots, C_{s}\right\}$ are conjugacy separable.

Proof First assume $F=\{1\}$, so $C_{i}=B_{i}$. In this case we shall see that most finitary fillings are fundamental groups of closed hyperbolic orbifolds. Thus they are virtually surface groups, hence conjugacy separable by a result of Martino [25, Thm. 3.7].

We define an obstacle set $S=S_{1} \cup \cdots \cup S_{s}$ in $P=\pi_{1}(\mathcal{O})$ as follows. Let $r$ be a large integer (to be determined later). Recall (see [40]) that each $B_{i}$ is either infinite cyclic or infinite dihedral. In the cyclic case, $B_{i}$ is generated by a single element $c$ of infinite order and we let $S_{i}:=\left\{c, c^{2}, \ldots, c^{r}\right\}$. In the dihedral case, $B_{i}$ is generated by two involutions $a, b$ and we let $S_{i}:=\left\{a, b, a b,(a b)^{2}, \ldots,(a b)^{r}\right\}$.

Any finite index normal subgroup $K_{i} \triangleleft B_{i}$ with $K_{i} \cap S_{i}=\emptyset$ is cyclic, generated by $c^{m_{i}}$ or $(a b)^{n_{i}}$ for some $m_{i}$ or $n_{i}$ larger than $r$. It follows that the quotient $\left.\pi_{1}(\mathcal{O}) /\left\langle K_{i} \mid i=1, \ldots, s\right\rangle\right\rangle^{\pi_{1}(\mathcal{O})}$ is the fundamental group of a closed orbifold $\mathcal{O}^{\prime}$, which is obtained from $\mathcal{O}$ by replacing each boundary component by a conical point (elliptic point, in the terminology of [40]) of order $m_{i}$ in the cyclic case, and by a dihedral point (corner reflector, in the terminology of [40]) of order $n_{i}$ in the dihedral case. 
We claim that $\mathcal{O}^{\prime}$ is hyperbolic if $r$ is large enough. By [40, Thm. 13.3.6], a 2-orbifold admits a hyperbolic structure if and only if its Euler characteristic is negative. The Euler characteristic $\chi\left(\mathcal{O}^{\prime}\right)$ can be computed by the following formula (cf. [40, 13.3.3], [36]):

$$
\chi\left(\mathcal{O}^{\prime}\right)=\chi(\mathcal{O})+\sum \frac{1}{m_{i}}+\sum \frac{1}{2 n_{i}} .
$$

Since $\chi(\mathcal{O})$ is negative, so is $\chi\left(\mathcal{O}^{\prime}\right)$ for $r$ large, and the claim follows. Defining $S$ using such an $r$, we deduce that most finitary fillings of $O$ are fundamental groups of closed hyperbolic orbifolds. This proves the proposition when $F=\{1\}$.

In the general case, we have to use Dehn fillings (Theorem 7.4). It is a standard fact [7, Thm. 7.11] that $O$ is hyperbolic relative to the family $\mathcal{H}=\left\{C_{1}, \ldots, C_{s}\right\}$ (these are non-conjugate maximal virtually cyclic subgroups of the hyperbolic group $O$ ). Consider the obstacle set $\bar{S}=\xi^{-1}(S) \cup S^{\prime}$, where $S$ is the set constructed above in $\pi_{1}(\mathcal{O})$ and $S^{\prime}$ is provided by Theorem 7.4 (applied to $O$ and $\mathcal{H}$ ).

Consider any collection of finite index normal subgroups $N_{i} \triangleleft C_{i}$ such that $N_{i} \cap \bar{S}=\emptyset$, $i=1, \ldots, s$, and set $N:=\left\langle\left\langle N_{i} \mid i=1, \ldots, s\right\rangle\right\rangle^{O}$. By Theorem 7.4 we have $N \cap C_{i}=N_{i}$ for each $i$, so it remains to check that the quotient $O^{\prime}=O / N$ is conjugacy separable.

Let $\varphi: O \rightarrow O^{\prime}$ denote the natural epimorphism. Then $O^{\prime}$ maps with finite kernel $\varphi(F)$ onto $\left.P / \xi(N) \cong P /\left\langle\xi\left(N_{i}\right) \mid i=1, \ldots, s\right\rangle\right\rangle^{P}$. Our choice of $\bar{S}$ guarantees that $\xi\left(N_{i}\right)$ does not meet the set $S$, so $P / \xi(N)$ is the fundamental group of a hyperbolic orbifold $\mathcal{O}^{\prime}$. The exact sequence $\{1\} \rightarrow \varphi(F) \rightarrow O^{\prime} \rightarrow \pi_{1}\left(\mathcal{O}^{\prime}\right) \rightarrow\{1\}$ implies that $O^{\prime}$ is virtually a surface group (see [25, Thm. 4.3]), hence it is conjugacy separable as above.

\subsection{Conclusion}

We prove Theorem 1.3, starting with a couple of lemmas. We first assume that no $P_{i}$ is virtually cyclic, postponing the general case to the next subsection.

Lemma 7.14 Consider the graph of groups $\Gamma^{\prime}$ constructed in Subsection 5, and assume, additionally, that the subgroups $P_{1}, \ldots, P_{k}$ are residually finite (and not virtually cyclic). Then $\Gamma^{\prime}$ satisfies Assumption 7.3. In particular, its fundamental group $G^{\prime}$ is residually finite (by Proposition 7.8).

Proof Recall that $\Gamma^{\prime}$ is bipartite, with $V_{1}=V_{E}$ and $V_{2}=V_{R} \cup V_{Q H}$. Vertex groups in $V_{1}$ are elementary, hence residually finite by assumption.

For $v \in V_{R}$, the vertex group $G_{v}^{\prime}$ is obtained by amalgamating $\mathbb{Z} \times C$ and the incident edge groups $G_{e}$ over a finite group $C$. Define $S=C \backslash\{1\}$ as the obstacle set. As in Remark 7.2, each finitary filling of $G_{v}^{\prime}$ with respect to the incident edge groups is an amalgam over $C$, with factors being finite or $\mathbb{Z} \times C$.

For $\mathrm{QH}$ vertices, residual finiteness (indeed, conjugacy separability) of finitary fillings follows from Lemma 7.13. The assumption that no $P_{i}$ is virtually cyclic guarantees that $\left\{C_{1}, \ldots, C_{s}\right\}$ is (up to conjugacy) the family of incident edge groups (see Subsection 4.2).

Relative hyperbolicity follows from standard combination theorems for relatively hyperbolic groups (cf. [10,30]) because vertex groups in $V_{2}$ are hyperbolic relative to incident edge groups: this was pointed out in the proof of Lemma 7.13 in the $\mathrm{QH}$ case, and in the rigid case this is a consequence of Definition 4.1 (as the graph $\mathcal{K}$ one can take the Bass-Serre tree associated to the splitting of $G_{v}^{\prime}, v \in V_{R}$, as an amalgam over $C$ discussed above). 
Lemma 7.15 Suppose that $G$ is a finitely generated group, and $N$ is a centerless normal subgroup of finite index in $G$.

(1) Some finite index subgroup $\operatorname{Out}_{0}(G) \leqslant \operatorname{Out}(G)$ is isomorphic to a quotient of a subgroup of $\operatorname{Out}(N)$ by a finite normal subgroup $L$.

(2) Let $\mathcal{P}$ be a finite family of subgroups in $G$ and let $\mathcal{Q}$ be a collection of representatives of $N$-conjugacy classes among $\left\{N \cap g \mathrm{Hg}^{-1} \mid H \in \mathcal{P}, g \in G\right\}$. Then some finite index subgroup of $\operatorname{Out}(G ; \mathcal{P})$ is isomorphic to a quotient of a subgroup of $\operatorname{Out}(N ; \mathcal{Q})$ by a finite normal subgroup. In particular, if $\operatorname{Out}(N ; \mathcal{Q})$ is residually finite then so is $\operatorname{Out}(G ; \mathcal{P})$.

Proof The first assertion is standard (see for instance [20, Lemma 5.4]). One defines $\operatorname{Aut}_{0}(G)$ as the set of automorphisms mapping $N$ to itself and acting as the identity on $G / N$, and $\operatorname{Out}_{0}(G)$ is its image in $\operatorname{Out}(G)$. Using the fact that $N$ is centerless, one shows that the natural map $\operatorname{Aut}_{0}(G) \rightarrow \operatorname{Aut}(N)$ is injective. The group $L$ comes from the action of inner automorphisms of $G$.

For 2, observe that automorphisms in $\operatorname{Aut}_{0}(G)$ preserving the set of conjugacy classes of groups in $\mathcal{P}$ also preserve the (finite) set of $N$-conjugacy classes of subgroups from $\mathcal{Q}$.

We can now prove Theorem 1.3 in the case when no peripheral subgroup is virtually cyclic:

Theorem 7.16 Let $G$ be a group hyperbolic relative to a family of proper finitely generated subgroups $\mathcal{P}=\left\{P_{1}, \ldots, P_{k}\right\}$. If $G$ is one-ended relative to $\mathcal{P}$, no $P_{i}$ is virtually cyclic, and every $P_{i}$ is residually finite, then $\operatorname{Out}(G ; \mathcal{P})$ is residually finite.

Proof Consider the canonical elementary JSJ tree $T$ of $G$ relative to the family $\mathcal{P}$, as in Subsection 4.2. If $T$ is trivial (a single vertex), then either $G$ is rigid or it maps onto the fundamental group of a closed hyperbolic 2-orbifold with finite kernel. In the former case $\operatorname{Out}(G ; \mathcal{P})$ is finite (see $[19$, Theorem 3.9]) and in the latter case $G$ contains a surface subgroup of finite index (see for instance [25, Thm. 4.3]). Therefore in this case $\operatorname{Out}(G)$ is residually finite by a combination of Grossman's theorem [15, Thm. 3] with Lemma 7.15.

Hence we can further assume that the canonical JSJ tree $T$ is non-trivial. Let us apply the construction of Section 5. By Lemma 5.3, a finite index $\operatorname{subgroup} \operatorname{Out}^{r}(G)$ of $\operatorname{Out}(G ; \mathcal{P})$ embeds into $\operatorname{Out}\left(G^{\prime}\right)$, where $G^{\prime}$ is the fundamental group of the bipartite graph of groups $\Gamma^{\prime}$.

If $T$ has at least one rigid vertex, then the group $G^{\prime}$ has infinitely many ends, because of the way we constructed $\Gamma^{\prime}$. Furthermore, $G^{\prime}$ is residually finite by Lemma 7.14. Therefore $\operatorname{Out}\left(G^{\prime}\right)$ is residually finite by $\left[28\right.$, Thm. 1.5], so its $\operatorname{subgroup} \operatorname{Out}^{r}(G)$, and also $\operatorname{Out}(G ; \mathcal{P})$, are residually finite.

Hence we can suppose that the JSJ decomposition of $G$ has no rigid vertices. In this case $G^{\prime}=G$ by construction, and $V_{2}=V_{Q H}$.

There are two cases. Assume, at first, that $E(G)=\{1\}$. Then, according to Proposition 7.13, the graph of groups $\Gamma^{\prime}$ satisfies all the assumptions of Proposition 7.11, which allows us to conclude that $\operatorname{Out}(G)$ (and, hence, $\operatorname{Out}(G ; \mathcal{P})$ ) is residually finite.

If $E(G) \neq\{1\}$, we shall deduce residual finiteness of $\operatorname{Out}(G ; \mathcal{P})$ from Lemma 7.15. As $E(G)$ is finite and $G$ is residually finite (by Lemma 7.14), there exists a finite index normal subgroup $N \triangleleft G$ such that $N \cap E(G)=\{1\}$. It is standard that $N$ is hyperbolic relative 
to the family $\mathcal{Q}$ described in the second part of Lemma 7.15 (this follows immediately from Definition 4.1). Note that groups in $\mathcal{Q}$ are finitely generated, residually finite, not virtually cyclic, and they are proper subgroups of $N$ since groups in $\mathcal{P}$ have infinite index in $G$ (indeed, each $P_{i} \in \mathcal{P}$ is almost malnormal in $G$ - see [31, Thm. 1.4]).

The group $E(N)$ is trivial because it is characteristic in $N$, hence it is contained in $E(G) \cap$ $N=\{1\}$. In particular, $N$ is centerless. Moreover, as pointed out in [18], it follows from Theorem IV.1.3 of [11] that $N$ is one-ended relative to $\mathcal{Q}$. Since $E(N)=\{1\}$, we know that $\operatorname{Out}(N ; \mathcal{Q})$ is residually finite by the previous case, and Lemma $7.15 \operatorname{implies}$ that $\operatorname{Out}(G ; \mathcal{P})$ is residually finite.

The arguments given above show the following facts, which may be of independent interest:

Corollary 7.17 Let $G$ be a group hyperbolic relative to a family $\mathcal{P}=\left\{P_{1}, \ldots, P_{k}\right\}$ of proper finitely generated residually finite groups, such that no $P_{i}$ is virtually cyclic. Suppose that $G$ is one-ended relative to $\mathcal{P}$.

If the canonical JSJ decomposition of $G$ over elementary subgroups relative to $\mathcal{P}$ has no rigid vertices, then $G$ is residually finite. Otherwise, a finite index $\operatorname{subgroup}$ of $\operatorname{Out}(G ; \mathcal{P})$ embeds into $\operatorname{Out}\left(G^{\prime}\right)$, where $G^{\prime}$ is a finitely generated residually finite group with infinitely many ends.

In all cases, $\operatorname{Out}(G ; \mathcal{P})$ virtually embeds into $\operatorname{Out}\left(G^{\prime}\right)$, where $G^{\prime}$ is a finitely generated residually finite relatively hyperbolic group.

\subsection{Allowing virtually cyclic $P_{i}$ 's}

We now prove Theorem 1.3 in general, allowing virtually cyclic peripheral subgroups. We may assume that all $P_{i}$ 's are infinite, since removing finite groups from $\mathcal{P}$ does not affect relative one-endedness.

The new phenomenon occurs at QH vertices of the canonical JSJ decomposition $\Gamma$. With the notations of Subsection 7.3, it is still true that incident edge groups of $G_{v}$ are preimages of fundamental groups of boundary components of $\mathcal{O}$, but there may now be boundary components $\mathcal{C}_{j}$ such that $C_{j}=\xi^{-1}\left(B_{j}\right)$ is not an incident edge group, but is conjugate to a group in $\mathcal{P}$.

In Section 5 we used groups $P M C G\left(G_{v}\right)$ and $P M C G^{\partial}\left(G_{v}\right)$, defined using incident edge groups of $\Gamma$. We must now replace $P M C G\left(G_{v}\right)$ with a subgroup, by requiring that automorphisms act on groups $C_{j}$ as above as inner automorphisms $\tau_{a_{j}}$ of $G_{v}$. The group $P M C G^{\partial}\left(G_{v}\right)$ is replaced by the preimage of this subgroup under $\pi_{v}$. We do not keep track of the $a_{j}$ 's; the corresponding $\mathcal{C}_{j}$ 's should be thought of as punctures rather than boundary components, in particular there is no twist near them (in the context of Subsection 3.2, isotopies are free on the components $\mathcal{C}_{j}$ ).

With this modification, all arguments given in Section 5, 6 and 7 go through. In Proposition 7.13, we define $\mathcal{H}$ using only the groups $C_{j}$ which are incident edge groups. The hyperbolic orbifold $\mathcal{O}^{\prime}$ may then have a non-empty boundary. In this case its fundamental group is virtually free, hence conjugacy separable by [13]. 


\subsection{Proof of Corollary 1.1}

Suppose that $G$ is hyperbolic relative to a family $\mathcal{P}=\left\{P_{1}, \ldots, P_{k}\right\}$ of virtually polycyclic groups. Without loss of generality we may assume that no $P_{i}$ is virtually cyclic (see Subsection 4.1). The result is true if $G$ is virtually polycyclic [43]. Otherwise, Theorem 1.3 applies since every $P_{i}$ is residually finite. To conclude, note that $\operatorname{Out}(G ; \mathcal{P})$ has finite index in $\operatorname{Out}(G)$ because groups in $\mathcal{P}$ are characterized (up to conjugacy) as maximal virtually polycyclic subgroups which are not virtually cyclic (see [27, Lemma 3.2] for a more general result).

\section{Residual $p$-finiteness for automorphism groups}

\subsection{Residual $p$-finiteness}

Given a prime $p$ and a group $G$, we will say that a subgroup $K \leqslant G$ has $p$-power index in $G$ if $|G: K|=p^{k}$ for some $k \geq 0$.

Remark 8.1 The intersection of two subgroups $H_{1}, H_{2}$ of $p$-power index is not necessarily of $p$-power index, but it is if $H_{1}$ and $H_{2}$ are normal (for then there is an embedding of $G /\left(H_{1} \cap H_{2}\right)$ into $\left.G / H_{1} \times G / H_{2}\right)$. In particular, if $G$ is finitely generated, any normal subgroup $H$ of $p$-power index contains one which is characteristic in $G$, namely the intersection of all subgroups of $G$ with the same index as $H$.

The collection of normal subgroups of $G$ of $p$-power index forms a basis of neighborhoods of the identity in $G$, giving rise to the pro-p topology on $G$. As in the case of residually finite groups, the pro- $p$ topology on $G$ is Hausdorff if and only if $G$ is residually $p$-finite: given $g \neq 1$, there is a homomorphism $\varphi$ from $G$ to a finite $p$-group such that $\varphi(g) \neq 1$ (by Remark 8.1, one may assume that $\operatorname{ker} \varphi$ is characteristic if $G$ is finitely generated).

Residual $p$-finiteness is a much more delicate condition than residual finiteness. It is still clearly stable under direct products, but in general it is not stable under semidirect products $(\mathbb{Z}$ is residually $p$-finite for any prime $p$, but it is easily checked that the Klein bottle group, the non-trivial semidirect product $\mathbb{Z} \rtimes \mathbb{Z}$, is not residually $p$-finite if $p>2$ ). Even more strikingly, for any given set of prime numbers $\Pi$, there exists a 3 -generated center-by-metabelian group which is residually $p$-finite if and only if $p \in \Pi-$ see [21].

Lemma 8.2 If $A$ has a residually $p$-finite normal subgroup $B$ of $p$-power index, then $A$ is residually $p$-finite.

Proof Take any $x \in A \backslash\{1\}$. Since $B$ is residually $p$-finite, there exists a $p$-power index normal subgroup $N \triangleleft B$ such that $x \notin N$. The intersection $H$ of all $A$-conjugates of $N$ is normal in $A$ and has $p$-power index in $B$ (see Remark 8.1), so $|A: H|=|A: B||B: H|$ is a power of $p$. Since $x \notin H$, we can conclude that $A$ is residually $p$-finite.

Remark 8.3 Combining Lemma 8.2 with an induction on the subnormal index, one can actually prove that any group containing a subnormal residually $p$-finite subgroup of $p$-power index is itself residually $p$-finite.

It is not difficult to see that not every $p$-power index subgroup of a group $G$ has to be closed in the pro- $p$ topology. In fact, a $p$-power index subgroup $K \leqslant G$ is closed in the pro- $p$ topology on $G$ if and only if $K$ is subnormal in $G$ (cf. [41, Lemma A.1]). 
Lemma 8.4 If $G$ is residually $p$-finite, and $N \triangleleft G$ is a finite normal subgroup, then $G / N$ is also residually $p$-finite.

Proof Indeed, since $G$ is residually $p$-finite, any finite subset of $G$ is closed in the pro- $p$ topology on $G$. Therefore $N$ is the intersection of $p$-power index normal subgroups of $G$, and so $G / N$ is residually $p$-finite.

For any prime $p$ and any group $H$, let $\operatorname{Aut}_{p}(H)$ be the subgroup of $\operatorname{Aut}(H)$ which consists of automorphisms that act trivially on the first mod- $p$ homology of $H$. Namely, let $K_{p}:=$ $[H, H] H^{p}$ be the verbal subgroup of $H$, which is the product of the derived subgroup $[H, H]$ and the subgroup $H^{p}$ generated by all the $p$-th powers of elements in $H$. Then

$$
\operatorname{Aut}_{p}(H)=\left\{\alpha \in \operatorname{Aut}(H) \mid \alpha\left(h K_{p}\right)=h K_{p} \text { for all } h \in H\right\} .
$$

If $H$ is finitely generated, then $K_{p}$ has finite index in $H$, therefore $\operatorname{Aut}_{p}(H)$ will have finite index in $\operatorname{Aut}(H)$. Observe also that all inner automorphisms are in $\operatorname{Aut}_{p}(H)$ because $H / K_{p}$ is abelian, and the group $\operatorname{Out}_{p}(H):=\operatorname{Aut}_{p}(H) / \operatorname{Inn}(H)$ has finite index in $\operatorname{Out}(H)$.

The following classical theorem of P. Hall will be useful (cf. [35, 5.3.2, 5.3.3]):

Lemma 8.5 If $H$ is a finite $p$-group, then $\operatorname{Aut}_{p}(H)$ is also a finite $p$-group.

The next statement was originally proved by L. Paris in [32, Thm 2.4]. We present an elementary proof based on Lemma 8.5.

Lemma 8.6 Let $H$ be a finitely generated residually $p$-finite group, for some prime $p$. Then $\operatorname{Aut}_{p}(H)$ is residually $p$-finite, hence $\operatorname{Aut}(H)$ is virtually residually $p$-finite.

Proof Consider any non-trivial automorphism $\alpha \in \operatorname{Aut}_{p}(H)$. Then there is $h_{0} \in H$ such that $\alpha\left(h_{0}\right) \neq h_{0}$. Since $H$ is residually $p$-finite, there exist a finite $p$-group $K$ and an epimorphism $\psi: H \rightarrow K$ with $\psi\left(\alpha\left(h_{0}\right)\right) \neq \psi\left(h_{0}\right)$ in $K$. As explained in Remark 8.1, one may assume that $\operatorname{ker} \psi$ is a characteristic subgroup of $H$. This implies that $\psi$ naturally induces a homomorphism $\varphi: \operatorname{Aut}(H) \rightarrow \operatorname{Aut}(K)$. Clearly, $\varphi\left(\operatorname{Aut}_{p}(H)\right) \subseteq \operatorname{Aut}_{p}(K)$, so the restriction $\varphi^{\prime}$ of $\varphi$ to $\operatorname{Aut}_{p}(H)$ gives a homomorphism from $\operatorname{Aut}_{p}(H)$ to $\operatorname{Aut}_{p}(K)$, where the latter is a finite $p$-group by Lemma 8.5. It remains to observe that $\varphi^{\prime}(\alpha)$ is non-trivial, because $\varphi^{\prime}(\alpha)\left(\psi\left(h_{0}\right)\right)=\psi\left(\alpha\left(h_{0}\right)\right) \neq \psi\left(h_{0}\right)$ by construction.

\subsection{Toral relatively hyperbolic groups}

In this section we will prove Theorem 1.5. The method is similar to the one used in Section 3.

Given a group $H$ with a fixed family of peripheral subgroups $C_{1}, \ldots, C_{s}, s \geq 1$, we can define $\operatorname{Aut}^{\partial}(H), P M C G^{\partial}(H)$ and $P M C G(H)$ as in Subsection 3.1. For any prime $p$, let $\operatorname{Aut}_{p}^{\partial}(H) \leqslant \operatorname{Aut}^{\partial}(H)$ consist only of those t-uples $\left(\alpha ; a_{1}, \ldots, a_{s}\right)$ for which $\alpha \in \operatorname{Aut}_{p}(H)$. In other words $\operatorname{Aut}_{p}^{\partial}(H)$ is the full preimage of $\operatorname{Aut}_{p}(H)$ under the natural projection $\operatorname{Aut}^{\partial}(H) \rightarrow \operatorname{Aut}(H)$. We also define $P M C G_{p}^{\partial}(H)$ as the image of $\operatorname{Aut}_{p}^{\partial}(H)$ in $P M C G^{\partial}(H)$, and $P M C G_{p}(H)$ will denote its image in $P M C G(H) \leqslant \operatorname{Out}(H)$.

Remark 8.7 If $H$ is finitely generated, then $P M C G_{p}^{\partial}(H)$ has finite index in $P M C G^{\partial}(H)$. 
Lemma 8.8 If $H$ is a finite $p$-group, then so is $P M C G_{p}^{\partial}(H)$. If $H$ is a finitely generated residually $p$-finite group, then $P M C G_{p}^{\partial}(H)$ is residually $p$-finite.

Proof $P M C G_{p}^{\partial}(H)$ embeds into $H^{s-1} \rtimes \operatorname{Aut}_{p}(H)$ (see Remark 3.3); this group is a finite $p$-group by Lemma 8.5. For the second assertion, argue as in Lemma 3.2, mapping $P M C G_{p}^{\partial}(H)$ to $P M C G_{p}^{\partial}(H / N)$ with $H / N$ a finite $p$-group.

Our next goal is Lemma 8.11 below, which is an analogue of Lemma 3.4. We need to prove two auxiliary statements first.

Lemma 8.9 Fundamental groups of closed hyperbolic surfaces are residually $p$-finite for all primes $p$.

Proof Let $\Sigma$ be a closed hyperbolic surface. Then $\pi_{1}(\Sigma)$ is residually free, except for the case when $\Sigma=\Sigma_{-1}$ is the closed non-orientable surface of genus 3 (and Euler characteristic -1 ), see $[6,4]$. Since free groups are residually $p$-finite for every prime $p$ (cf. $[35,6.1 .9]$ ), the lemma follows for all $\Sigma \neq \Sigma_{-1}$. On the other hand, for any prime $p$, there is a normal cover of degree $p$ of $\Sigma_{-1}$ (because the abelianization of $\pi_{1}\left(\Sigma_{-1}\right)$ is isomorphic to $\mathbb{Z}^{2} \times \mathbb{Z} / 2 \mathbb{Z}$ ). This cover is a surface of higher genus, so its fundamental group is residually $p$-finite by the previous argument. Hence $\pi_{1}\left(\Sigma_{-1}\right)$ is residually $p$-finite by Lemma 8.2.

Lemma 8.10 Let $p$ be a prime, and $n$ be a power of $p$. Let $\Sigma_{n}$ be a closed hyperbolic 2-orbifold whose singularities are cone points of order $n$. Then $O_{n}:=\pi_{1}\left(\Sigma_{n}\right)$ is residually p-finite.

Proof Let $\Sigma$ be a compact surface obtained by removing a neighborhood of each conical point. We may map $\pi_{1}(\Sigma)$ to a finite $p$-group so that the fundamental group of every boundary component has image of order exactly $n$ : if $\Sigma$ has only one boundary component, this follows from [39, Lemma 1] (see also [25, Lemma 4.1]); if there are more, the fundamental group of each boundary component is a free generator of $\pi_{1}(\Sigma)$, and we map $\pi_{1}(\Sigma)$ to $H_{1}\left(\pi_{1}(\Sigma), \mathbb{Z} / n \mathbb{Z}\right)$, its abelianization $\bmod n$.

The corresponding normal covering of $\Sigma$ extends to a covering of $\Sigma_{n}$ by a closed surface, because its restriction to every component of $\partial \Sigma$ has degree exactly $n$. The fundamental group of this surface is residually $p$-finite by Lemma 8.9. Its index in $O_{n}$ is a power of $p$, so $O_{n}$ is residually $p$-finite by Lemma 8.2.

Now suppose, as in Subsection 3.2, that $H$ is the fundamental group of a compact surface $\Sigma$ with negative Euler characteristic and $s \geq 1$ boundary components. Let $C_{1}, \ldots, C_{s}$ be the fundamental groups of these components, considered as subgroups of $H$. Let $\mathcal{T}_{H} \leqslant P M C G^{\partial}(H)$ be the corresponding group of twists. Note that $\mathcal{T}_{H} \subseteq P M C G_{p}^{\partial}(H)$ for any prime $p$. We have the following analogue of Lemma 3.4:

Lemma 8.11 Let $p$ be a prime. Then the quotient $P M C G_{p}^{\partial}(H) / n \mathcal{T}_{H}$ is residually $p$-finite for every sufficiently large power $n$ of $p$.

Proof The proof is similar to that of Lemma 3.4, using $P M C G_{p}$ instead of $P M C G$. The kernel of $\theta: \mathcal{T}_{H} \rightarrow \mathcal{T}_{O_{n}}$ is $n \mathcal{T}_{H}$, and we need to know that $P M C G_{p}(H)$ and $P M C G_{p}^{\partial}\left(O_{n}\right)$ are residually $p$-finite.

Clearly $P M C G_{p}(H) \leqslant \operatorname{Out}_{p}(H)$, which is residually $p$-finite by a result of L. Paris [32, Thm. 1.4] since $H$ is a free group. On the other hand, the group $P M C G_{p}^{\partial}\left(O_{n}\right)$ is residually $p$-finite by Lemmas 8.10 and 8.8 . 
We also need to consider abelian groups. Let $A$ be a free abelian group of finite rank with a chosen family of subgroups $C_{1}, \ldots, C_{s}$. For any prime $p$, consider the corresponding groups $\operatorname{Aut}_{p}^{\partial}(A), P M C G_{p}^{\partial}(A)$, and the normal subgroup $\mathcal{T}_{A} \triangleleft P M C G_{p}^{\partial}(A)$, defined as in Subsection 3.1. Note that $\mathcal{T}_{A}$ is naturally isomorphic to the quotient of $A^{s}$ by its diagonal subgroup, hence to $A^{s-1}$.

Lemma 8.12 Let $p$ be a prime. The quotient $P M C G_{p}^{\partial}(A) / n \mathcal{T}_{A}$ is residually $p$-finite for every power $n$ of $p$.

Proof Consider the following commutative diagram of short exact sequences:

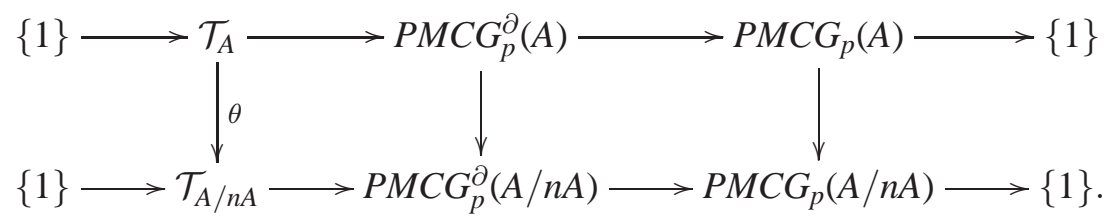

The map $\theta: \mathcal{T}_{A} \rightarrow \mathcal{T}_{A / n A}$ sends $A^{s-1}$ to $(A / n A)^{s-1}$, so its kernel is $n \mathcal{T}_{A}$ and the proof is reduced to showing that $P M C G_{p}(A)$ and $P M C G_{p}^{\partial}(A / n A)$ are residually $p$-finite. Now $P M C G_{p}(A) \leqslant \operatorname{Out}_{p}(A)=\operatorname{Aut}_{p}(A)$ is residually $p$-finite by Lemma 8.6, and $P M C G_{p}^{\partial}(A / n A)$ is a finite $p$-group by Lemma 8.8 .

Lemma 8.13 (cf. Lemma 3.6) Consider a finite set $V$ and groups $P_{v}, v \in V$, with normal subgroups $T_{v}$ free abelian of finite ranks. Let $P=\prod_{v \in V} P_{v}$ and $T=\prod_{v \in V} T_{v} \leqslant P$ be their direct products.

Suppose that $p$ is a prime number and $Z \leqslant T$ is a subgroup such that $T / Z$ contains no $q$-torsion if $q \neq p$ is a prime. If $P_{v} / n T_{v}$ is residually $p$-finite for all $v \in V$ and for every sufficiently large power $n$ of $p$, then $Z$ is closed in the pro- $p$ topology of $P$. In particular, if $Z$ is normal in $P$, then $P / Z$ is residually $p$-finite.

Proof This is similar to the proof of Lemma 3.6. One first proves the result when $Z$ has $p$-power index in $T$. In the general case, $T / Z$ being residually $p$-finite guarantees that $Z$ is the intersection of (normal) subgroups of $p$-power index in $T$.

We are now ready to prove the main theorem of this section.

Theorem 8.14 If some finite index subgroup of $G$ is a one-ended toral relatively hyperbolic group, then $\operatorname{Out}(G)$ is virtually residually $p$-finite for any prime $p$.

Proof First suppose that $G$ itself is torsion-free and hyperbolic relative to a family $\mathcal{P}=$ $\left\{P_{1}, \ldots, P_{k}\right\}$ of free abelian groups of finite rank. As in the proof of Corollary 1.1, we can assume that no $P_{i} \in \mathcal{P}$ is cyclic, and restrict to $\operatorname{Out}(G ; \mathcal{P})$ because it has finite index in $\operatorname{Out}(G)$. Consider the canonical JSJ tree $T$ relative to $\mathcal{P}$ over abelian groups as in Subsection 4.2.

If $T$ consists of a single point then either $G$ is rigid, or $G$ is the fundamental group of a closed hyperbolic surface $\Sigma$, or $G$ is a finitely generated free abelian group. In the first case $\operatorname{Out}(G)$ is finite (see [19] for instance). In the second case, if $\Sigma$ is orientable then $\operatorname{Out}_{p}(G)$ is residually $p$-finite by [32, Thm. 1.4], and if $\Sigma$ is non-orientable, then it possesses an orientable cover $\Sigma^{\prime}$ of degree 2 . Since the group $\operatorname{Out}\left(\pi_{1}\left(\Sigma^{\prime}\right)\right)$ is virtually residually $p$-finite by [32, Thm. 1.4], and $\pi_{1}\left(\Sigma^{\prime}\right)$ is a centerless normal subgroup of finite index of $G$, we can use Lemmas 7.15 and 8.4 to conclude that $\operatorname{Out}(G)$ is virtually residually $p$-finite. Finally, 
if $G$ is a free abelian group of finite $\operatorname{rank}$, then $\operatorname{Out}_{p}(G)=\operatorname{Aut}_{p}(G)$ is residually $p$-finite by Lemma 8.6.

Thus we can suppose that the tree $T$ is non-trivial. In this case we know (cf. Lemma 5.2 and Remark 8.7) that $\operatorname{Out}_{p}^{r}(G)$, the image by $\lambda_{E, Q H}$ of $\prod_{v \in V_{E} \cup V_{Q H}} P M C G_{p}^{\partial}\left(G_{v}\right)$, has finite index in $\operatorname{Out}(G ; \mathcal{P})$. We apply Lemma 8.13 to $P=\prod_{v \in V_{E} \cup V_{Q H}} P M C G_{p}^{\partial}\left(G_{v}\right)$, with $T_{v}=\mathcal{T}_{v}$.

We know that each $P M C G_{p}^{\partial}\left(G_{v}\right) / n \mathcal{T}_{v}$ is residually $p$-finite for $n$ a large power of $p$, by Lemmas 8.11 and 8.12. The quotient of $\prod_{v \in V_{E} \cup V_{O H}} \mathcal{T}_{v}$ by $Z=\operatorname{ker} \lambda_{E, Q H}$ is the whole group

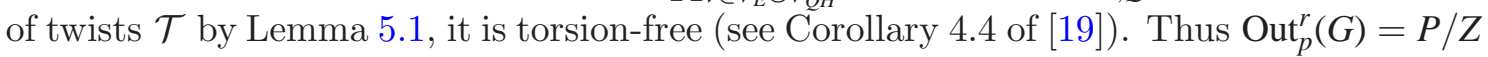
is residually $p$-finite by Lemma 8.13. It has finite $\operatorname{index} i n \operatorname{Out}(G)$, so $\operatorname{Out}(G)$ is virtually residually $p$-finite.

Now suppose that $G$ contains a toral relatively hyperbolic group $G_{0}$ with finite index. We may assume that $G_{0}$ is normal. If $G_{0}$ is abelian, then $\operatorname{Out}(G)$ is contained in some $G L(n, \mathbb{Z})$ by [43], so it is virtually residually $p$-finite by Lemma 8.6 (as $G L(n, \mathbb{Z}) \cong \operatorname{Aut}\left(\mathbb{Z}^{n}\right)$ ). Otherwise $G_{0}$ has trivial center and we apply Lemmas 7.15 and 8.4.

\subsection{Groups with infinitely many ends}

In this subsection we prove Theorem 1.6: if $G$ is a finitely generated group with infinitely many ends, and $G$ is virtually residually $p$-finite for some prime number $p$, then $\operatorname{Out}(G)$ is virtually residually $p$-finite. The argument will use the following "pro- $p$ " analogue of Lemma 7.12:

Lemma 8.15 Let $p$ be a prime. Given a finitely generated group $G$ and $\alpha \in \operatorname{Aut}_{p}(G)$, suppose that there is a homomorphism $\psi: G \rightarrow K$ with $K$ a finite p-group such that $\psi(g)$ is not conjugate to $\psi(\alpha(g))$ in $K$. Then there is a homomorphism $\phi: \operatorname{Out}_{p}(G) \rightarrow L$ with $L$ a finite $p$-group such that $\phi(\hat{\alpha}) \neq 1$ in $L$, where $\hat{\alpha}$ denotes the image of $\alpha$ in $\operatorname{Out}_{p}(G)$.

Proof The proof is almost identical to the proof of Lemma 7.12, except one needs to use Aut ${ }_{p}$ and $\mathrm{Out}_{p}$ instead of Aut and Out, together with the fact that $\mathrm{Out}_{p}(H)$ is a finite $p$-group for any finite $p$-group $H$, which immediately follows from Lemma 8.5.

Proof of Theorem 1.6 Recall that by Stallings' theorem for groups with infinitely many ends [38], the group $G$ splits as an amalgamated product or as an HNN-extension over a finite subgroup $C \leqslant G$. Since $G$ is virtually residually $p$-finite we can find a finite index normal subgroup $H \triangleleft G$ such that $H \cap C=\{1\}$ and $H$ is residually $p$-finite. It follows from the generalized Kurosh theorem (cf. [11, I.7.7] or [9, Thm. 8.27]) that $H=A * B$, where $A$ and $B$ are non-trivial finitely generated residually $p$-finite groups. Note that $H$ has trivial center (as does any non-trivial free product), and so by Lemmas 7.15 and 8.4 it is enough to prove that $\operatorname{Out}(H)$ is virtually residually $p$-finite.

Observe that $H$ is hyperbolic relative to $\{A, B\}$ and consider any automorphism $\alpha \in$ $\operatorname{Aut}_{p}(H) \backslash \operatorname{Inn}(H)$. Again, since $H$ splits as a non-trivial free product, $H$ contains no nontrivial finite normal subgroups, hence $E(H)=\{1\}$. Therefore, according to Lemma 7.9, there exists $g \in H$ such that both $g$ and $h:=\alpha(g)$ are loxodromic in $H$ and $g$ is not commensurable with $h$ in $H$. Since $A$ and $B$ are residually $p$-finite, applying Lemma 7.10, we can find normal subgroups $A^{\prime} \triangleleft A$ and $B^{\prime} \triangleleft B$ such that $A_{1}:=A / A^{\prime}$ and $B_{1}:=B / B^{\prime}$ are finite $p$-groups and the images of $g$ and $h$ are non-commensurable in the free product $H_{1}:=A_{1} * B_{1}$. 
We claim that $H_{1}$ is conjugacy $p$-separable, i.e., given two non-conjugate $h, h^{\prime} \in H$ there exist a finite $p$-group $K$ and a homomorphism $\xi: H_{1} \rightarrow K$ such that $\xi(h)$ is not conjugate to $\xi\left(h^{\prime}\right)$ in $K$. Indeed, by the Kurosh subgroup theorem, the kernel of the natural map $A_{1} * B_{1} \rightarrow A_{1} \times B_{1}$ is free, thus $H_{1}$ is an extension of a finitely generated free group by the finite $p$-group $A_{1} \times B_{1}$. Hence, by a theorem of E. Toinet [41, Thm. 1.7], $H_{1}$ is conjugacy $p$-separable (in fact, the full strength of Toinet's result is not needed here: conjugacy $p$-separability of free products of finite $p$-groups can be derived from the conjugacy $p$-separability of the free group via a short argument, similar to the one used by V. Remeslennikov in [33, Thm. 2]).

Let $\eta: H \rightarrow H_{1}$ denote the natural homomorphism with $\operatorname{ker} \eta=\left\langle\left\langle A^{\prime}, B^{\prime}\right\rangle\right\rangle^{H}$. Let $\psi:=\xi \circ \eta$ : $H \rightarrow K$. Then $\psi(h)=\psi(\alpha(g))$ is not conjugate to $\psi(g)$ in $K$ by construction. Therefore by Lemma 8.15 there is a finite $p$-group $L$ and a homomorphism $\phi: \operatorname{Out}_{p}(H) \rightarrow L$ such that $\phi(\hat{\alpha}) \neq 1$ in $L$, where $\hat{\alpha}$ is the image of $\alpha$ in $\operatorname{Out}_{p}(H)$. Thus we have shown that $\operatorname{Out}_{p}(H)$ is residually $p$-finite. Since $H$ is finitely generated, $\left|\operatorname{Out}(H): \operatorname{Out}_{p}(H)\right|<\infty$, and $\operatorname{so} \operatorname{Out}(H)$ is virtually residually $p$-finite, as required.

Before concluding let us discuss one application of Theorem 1.6. In a recent paper [3] Aschenbrenner and Friedl proved that the fundamental group of any compact 3-manifold $M$ is residually $p$-finite for all but finitely many primes $p$. Recalling Lubotzky's theorem [24, Prop. 2], they derived that $\operatorname{Aut}\left(\pi_{1}(M)\right)$ is virtually residually $p$-finite and mentioned that the similar fact for $\operatorname{Out}\left(\pi_{1}(M)\right)$ is not yet known. Theorem 1.6 implies that if a compact orientable 3-manifold $M$ is not irreducible, then $\operatorname{Out}\left(\pi_{1}(M)\right)$ is virtually residually $p$-finite (for all but finitely many primes $p$ ). Indeed, since $M$ is not irreducible, either it is $\mathbb{S}^{2} \times \mathbb{S}^{1}$ or it decomposes into a connected sum of prime manifolds. In the former case $\pi_{1}(M) \cong \mathbb{Z}$, and in the latter case $\pi_{1}(M)$ splits as a non-trivial free product. Thus either $\operatorname{Out}\left(\pi_{1}(M)\right)$ is finite, or $\pi_{1}(M)$ has infinitely many ends, and so $\operatorname{Out}\left(\pi_{1}(M)\right)$ is virtually residually $p$-finite for all but finitely many $p$ 's by Theorem 1.6 (using the result of Aschenbrenner and Friedl [3] mentioned above). Therefore, in order to prove that $\operatorname{Out}\left(\pi_{1}(M)\right)$ is virtually residually $p$-finite for all compact orientable 3-manifolds $M$, it is enough to consider only irreducible manifolds.

As a finishing remark, one can recall the theorem of Rhemtulla [34] stating that if a group is residually $p$-finite for infinitely many primes $p$, then it is bi-orderable. Unfortunately our methods do not allow to $\operatorname{deduce}$ that $\operatorname{Out}(G)$ has a single finite index subgroup which is residually $p$-finite for infinitely many $p$ 's. This is because we rely on Lemma 8.5, requiring one to pass to the subgroup $\operatorname{Out}_{p}(G)$, the index of which generally depends on the prime $p$.

\section{References}

[1] E. Alibegović, A combination theorem for relatively hyperbolic groups. Bull. London Math. Soc. 37 (2005), no. 3, 459-466.

[2] R.B.J.T. Allenby, G. Kim, C.Y. Tang, Residual finiteness of outer automorphism groups of certain pinched 1-relator groups. J. Algebra 246 (2001), no. 2, 849-858.

[3] M. Aschenbrenner, S. Friedl, 3-manifold groups are virtually residually p. Mem. Amer. Math. Soc. 225 (2013), no. 1058, viii+100 pp.

[4] B. Baumslag, Residually free groups. Proc. London Math. Soc. (3) 17 (1967), pp. 402-418.

[5] G. Baumslag, Automorphism groups of residually finite groups. J. London Math. Soc. 38 (1963), 117-118. 
[6] G. Baumslag, On generalised free products. Math. Z. 78 (1962), pp. 423-438.

[7] B.H. Bowditch, Relatively hyperbolic groups. Internat. J. Algebra Comput. 22 (2012), no. 3, 1250016, 66 pp.

[8] I. Bumagin, D.T. Wise, Every group is an outer automorphism group of a finitely generated group. J. Pure Appl. Algebra 200 (2005), no. 1-2, 137-147.

[9] D.E. Cohen, Combinatorial group theory: a topological approach. London Mathematical Society Student Texts, 14. Cambridge University Press, Cambridge, 1989. x+310 pp.

[10] F. Dahmani, Combination of convergence groups. Geom. \& Top. 7 (2003), 933-963.

[11] W. Dicks, M.J. Dunwoody, Groups acting on graphs. Cambridge Studies in Advanced Mathematics, 17. Cambridge University Press, Cambridge, 1989. xvi+283 pp.

[12] J.L. Dyer, Separating conjugates in amalgamated free products and HNN extensions. $J$. Austral. Math. Soc. Ser. A 29 (1980), no. 1, 35-51.

[13] J.L. Dyer, Separating conjugates in free-by-finite groups. J. London Math. Soc. (2) 20 (1979), no. 2, 215-221.

[14] L. Funar, Two questions on mapping class groups. Proc. Amer. Math. Soc. 139 (2011), 375-382.

[15] E.K. Grossman, On the residual finiteness of certain mapping class groups. J. London Math. Soc. (2) 9 (1974/75), 160-164.

[16] D. Groves, J.F. Manning, Dehn filling in relatively hyperbolic groups. Israel J. Math. 168 (2008), 317-429.

[17] V. Guirardel, G. Levitt, JSJ decompositions: definitions, existence, uniqueness, II. Compatibility and acylindricity. arXiv: 1002.4564

[18] V. Guirardel, G. Levitt, McCool groups of toral relatively hyperbolic groups. In preparation.

[19] V. Guirardel, G. Levitt, Splittings and automorphisms of relatively hyperbolic groups. Groups Geom. Dyn., to appear. arXiv:1212.1434.

[20] V. Guirardel, G. Levitt, The outer space of a free product. Proc. London Math. Soc. 94 (2007), no. 3, 695-714.

[21] B. Hartley, On residually finite p-groups. Symposia Mathematica, Vol. XVII (Convegno sui Gruppi Infiniti, INDAM, Roma, 1973), pp. 225-234. Academic Press, London, 1976.

[22] C. Hruska, Relative hyperbolicity and relative quasiconvexity for countable groups. Algebr. Geom. Topol. 10 (2010), no. 3, 1807-1856.

[23] G. Levitt, Automorphisms of hyperbolic groups and graphs of groups. Geom. Dedicata 114 (2005), 49-70.

[24] A. Lubotzky, Normal automorphisms of free groups. J. Algebra 63 (1980), no. 2, 494-498.

[25] A. Martino, A proof that all Seifert 3-manifold groups and all virtual surface groups are conjugacy separable. J. Algebra 313 (2007), no. 2, 773-781.

[26] V. Metaftsis, M. Sykiotis, On the residual finiteness of outer automorphisms of relatively hyperbolic groups. J. Pure Appl. Algebra 214 (2010), no. 8, 1301-1305.

[27] A. Minasyan, D. Osin, Fixed subgroups of automorphisms of relatively hyperbolic groups. Q. J. Math. 63 (2012), no. 3, pp. 695-712.

[28] A. Minasyan, D. Osin, Normal automorphisms of relatively hyperbolic groups. Trans. Amer. Math. Soc. 362 (2010), no. 11, 6079-6103.

[29] D.V. Osin, Peripheral fillings of relatively hyperbolic groups, Invent. Math. 167 (2007), no. 2, 295-326.

[30] D.V. Osin, Relative Dehn functions of amalgamated products and HNN-extensions. Topological and asymptotic aspects of group theory, 209-220, Contemp. Math., 394, Amer. Math. Soc., Providence, RI, 2006.

[31] D.V. Osin, Relatively hyperbolic groups: intrinsic geometry, algebraic properties, and algorithmic problems, Mem. Amer. Math. Soc. 179 (2006), no. 843. 
[32] L. Paris, Residual $p$ properties of mapping class groups and surface groups. Trans. Amer. Math. Soc. 361 (2009), no. 5, 2487-2507.

[33] V.N. Remeslennikov, Finite approximability of groups with respect to conjugacy (Russian). Sibirsk. Mat. Ž. 12 (1971), 1085-1099. English translation in Siberian Math. J. 23 (1971), 783-792.

[34] A.H. Rhemtulla, Residually $F_{p}$-groups, for many primes $p$, are orderable. Proc. Amer. Math. Soc. 41 (1973), 31-33.

[35] D.J.S. Robinson, A course in the theory of groups. Second edition. Graduate Texts in Mathematics, 80. Springer-Verlag, New York, 1996. xviii+499 pp.

[36] P. Scott, The geometries of 3-manifolds. Bull. London Math. Soc. 15 (1983), no. 5, 401-487.

[37] J.-P. Serre, Arbres, amalgames, $\mathrm{SL}_{2}$, Astérisque, no. 46. Société Mathématique de France, Paris, 1977. 189 pp.

[38] J. Stallings, Group theory and three-dimensional manifolds. A James K. Whittemore Lecture in Mathematics given at Yale University, 1969. Yale Mathematical Monographs, 4. Yale University Press, New Haven, Conn.-London, 1971. v+65 pp.

[39] P.F. Stebe, Conjugacy separability of certain free products with amalgamation. Trans. Amer. Math. Soc. 56 (1971), 119-129.

[40] W. Thurston, The geometry and topology of three-manifolds, Princeton lecture notes, 1980. http://library.msri.org/books/gt3m/

[41] E. Toinet, Conjugacy p-separability of right-angled Artin groups and applications. Groups Geom. Dyn. 7 (2013), no. 3, 751-790.

[42] B.A.F. Wehrfritz, Infinite linear groups. An account of the group-theoretic properties of infinite groups of matrices. Ergebnisse der Matematik und ihrer Grenzgebiete, Band 76. Springer-Verlag, New York-Heidelberg, 1973. xiv+229 pp.

[43] B.A.F. Wehrfritz, Two remarks on polycyclic groups. Bull. London Math. Soc. 26 (1994), no. $6,543-548$.

Laboratoire de Mathématiques Nicolas Oresme, Université de Caen et CNRS (UMR 6139), BP 5186, 14032 CAEN Cedex 5, France

Mathematical Sciences, University of Southampton, Highfiled Southampton SO17 1BJ, UK

levitt@unicaen.fr, aminasyan@gmail.com 